\title{
Pre-metastatic niches: organ-specific homes for metastases
}

\author{
Héctor Peinado ${ }^{1,2 *}$, Haiying Zhang ${ }^{1 *}$, Irina R. Matei ${ }^{*}$, Bruno Costa-Silva ${ }^{1,3}$, \\ Ayuko Hoshino', Goncalo Rodrigues ${ }^{1,4}$, Bethan Psaila ${ }^{5}$, Rosandra N. Kaplan ${ }^{6}$, \\ Jacqueline F. Bromberg7, Yibin Kang ${ }^{8,9}$, Mina J. Bissell'10, Thomas R. Cox ${ }^{11}$, \\ Amato J. Giaccia' ${ }^{12}$, Janine T. Erler's, Sachie Hiratsuka ${ }^{14}$, Cyrus M. Ghajar ${ }^{15}$ \\ and David Lyden ${ }^{1,16}$
}

\begin{abstract}
It is well established that organs of future metastasis are not passive receivers of circulating tumour cells, but are instead selectively and actively modified by the primary tumour before metastatic spread has even occurred. Sowing the 'seeds' of metastasis requires the action of tumour-secreted factors and tumour-shed extracellular vesicles that enable the 'soil' at distant metastatic sites to encourage the outgrowth of incoming cancer cells. In this Review, we summarize the main processes and new mechanisms involved in the formation of the pre-metastatic niche.
\end{abstract}

Disseminated tumour cells (DTCS). Thought to originate from CTCs that reach distant organs and survive in these new distant

microenvironments

Tumour-secreted factors Also known as the tumour secretome. The totality of factors released by tumour cells into their immediate environment or into the systemic circulation. They include growth factors hormones, cytokines, chemokines and extracellular matrix components, as well as extracellular vesicles.
Correspondence to D.L. Children's Cancer and Blood Foundation Laboratories, Departments of Pediatrics, and Cell and Developmental Biology, Drukier Institute for Children's Health, Meyer Cancer Center, Weill Cornell Medicine, New York, New York 10021, USA dc12001@med.cornell.edu
A pivotal discovery by Stephen Paget in 1889 (REF. 1) postulated that metastasis is dependent on the interactions between 'seeds' (or the cancer cells) and the 'soil' (or the host microenvironment). Paget's theory was challenged in the 1930s by James Ewing, who advocated that metastatic dissemination could be explained solely by the dynamics of haematogenous flow ${ }^{2}$ (FIG. 1). Ewing's perspective became the prevalent viewpoint until Isaiah Fidler's research in the 1970s demonstrated that, although the mechanical properties of blood flow were important, successful metastatic colonization could occur only at certain organ sites $^{3,4}$. In addition to strengthening Paget's theory, Fidler's findings reignited interest in the question that first captivated Paget ${ }^{3,5}$ : why do tumour cells emerge only as disseminated tumour cells (DTCs) within specific organs? Is metastatic seeding monoclonal or polyclonal in nature? Moreover, does metastatic seeding occur only directly from the primary tumour or is secondary seeding from one metastatic organ to another also a biologically relevant event? This organ specificity observed in metastasis is known as organotropism and remains one of the most intriguing unanswered questions in cancer research.

Additional fundamental discoveries revealed that tumours induce the formation of microenvironments in distant organs that are conducive to the survival and outgrowth of tumour cells before their arrival at these sites ${ }^{6-9}$. These predetermined microenvironments are termed 'pre-metastatic niches' (PMNs) ${ }^{7}$ (FICS 1,2). Since the existence of the PMN was first demonstrated, numerous studies have identified various molecules that regulate its stepwise evolution, highlighting the complex molecular and cellular changes that occur in the PMN to support future metastatic tumour growth $^{6,10,11}$ (FIG. 2). PMNs are the result of the combined systemic effects of tumour-secreted factors and tumourshed extracellular vesicles (EVs) that promote a temporal sequence of events during the evolution of PMNs. Vascular leakiness is the earliest event in this sequence, followed by the alteration of local resident cells, such as fibroblasts, and the recruitment of non-resident cells, such as bone marrow-derived cells (BMDCs), to these PMNs, subsequently attracting circulating tumour cells (CTCs) ${ }^{6,7,12}$. Although congruent with both Paget's and Ewing's theories, the concept of the PMN is unique as it proposes that the primary tumour preconditions specific organ sites for future metastatic disease (that is, before CTC arrival) via tumour-derived factors. Therefore, in contrast to the metastatic niche, which is initiated and shaped upon CTC arrival, the PMN represents an abnormal, tumour growth-favouring microenvironment devoid of cancer cells.

In addition to the contribution of tumour-secreted factors to PMN formation, there are other tumourindependent pathological and physiological processes involved, such as the effects of surgery, infection and ageing (that is, the ageing bone marrow), which alter the local milieu and help to create a microenvironment that is sufficiently receptive to colonization by CTCs ${ }^{13-15}$. It has become clear that extracellular matrix (ECM) remodelling is crucial for establishing the PMN (FIG. 2). Moreover, PMNs are probable sites of immune deregulation, owing to the presence of a pro-tumorigenic, 


\author{
Author addresses \\ ${ }^{1}$ Children's Cancer and Blood Foundation Laboratories, Departments of Pediatrics, and Cell \\ and Developmental Biology, Drukier Institute for Children's Health, Meyer Cancer Center, \\ Weill Cornell Medicine, New York, New York 10021, USA. \\ ${ }^{2}$ Microenvironment and Metastasis Group, Department of Molecular Oncology, Spanish \\ National Cancer Research Center (CNIO), Madrid 28029, Spain. \\ 35ystems Oncology Group, Champalimaud Research, Champalimaud Centre for the \\ Unknown, Avenida Brasília, Doca de Pedrouços, 1400-038 Lisbon, Portugal. \\ ${ }^{4}$ Graduate Program in Areas of Basic and Applied Biology, Abel Salazar Biomedical Sciences \\ Institute, University of Porto, 4099-003 Porto, Portugal. \\ ${ }^{5}$ Centre for Haematology, Department of Medicine, Hammersmith Hospital, Imperial \\ College London, London W12 0HS, UK. \\ ${ }^{6}$ Center for Cancer Research, Pediatric Oncology Branch, National Cancer Institute, \\ National Institutes of Health, Building 10-Hatfield CRC, Room 1-3940, Bethesda, \\ Maryland 20892, USA. \\ ${ }^{7}$ Department of Medicine, Memorial Sloan Kettering Cancer Center, New York, New York \\ 10065, USA. \\ ${ }^{8}$ Department of Molecular Biology, Princeton University, Princeton, New Jersey 08544, USA. \\ ${ }^{9}$ Rutgers Cancer Institute of New Jersey, New Brunswick, New Jersey 08903, USA. \\ ${ }^{10}$ Biological Systems and Engineering Division, Lawrence Berkeley National Laboratory, \\ Berkeley, California 94720, USA. \\ ${ }^{11}$ The Garvan Institute of Medical Research and The Kinghorn Cancer Centre, Cancer \\ Division, St Vincent's Clinical School, Faculty of Medicine, University of New South Wales, \\ Sydney, NSW 2010, Australia. \\ ${ }^{12}$ Department of Radiation Oncology, Stanford University, Stanford, California 94305, USA. \\ ${ }^{13}$ Biotech Research and Innovation Centre (BRIC), University of Copenhagen (UCPH), \\ Copenhagen 2200, Denmark. \\ ${ }^{14}$ Department of Pharmacology, Tokyo Women's Medical University School of Medicine, \\ 8-1 Kawada-cho, Tokyo 162-8666, Japan. \\ ${ }^{15}$ Public Health Sciences Division/Translational Research Program, Fred Hutchinson Cancer \\ Research Center, Seattle, Washington 98109, USA. \\ ${ }^{16}$ Department of Pediatrics, Memorial Sloan Kettering Cancer Center, New York, \\ New York 10065, USA. \\ *These authors contributed equally to this work
}

Extracellular vesicles

(EVs). A heterogeneous population of membranesurrounded structures released by cells into the intercellular space and the circulation. Their sizes range from $30 \mathrm{~nm}$ to $5 \mu \mathrm{m}$ in diameter and they include exosomes (typically

30-150 nm), microvesicles (150-1,000 nm) and apoptotic bodies $(1-5 \mu \mathrm{m})$.

Vascular leakiness Loss of vascular integrity resulting in increased permeability of vessels to macromolecules and cells that normally face resistance or do not cross endothelial barriers.

Circulating tumour cells (CTCS). Rare cells shed by solid tumours into the systemic circulation at an estimated frequency of 1:500,000$1: 1,000,000$ circulating cells.

Metastatic niche Microenvironment in distant organs that supports the survival and outgrowth of tumour cells. inflammatory milieu induced by tumour-secreted factors, which creates immunosuppression and coagulation disorders ${ }^{6,10,16}$ (FIG. 2).

In this Review, we summarize the progression of PMN formation. We discuss the main mechanisms involved in PMN generation and their impact on host cells in pre-metastatic organs. We also discuss a novel concept: the generation of specialized microenvironments, named 'sleepy niches', where tumour cells could survive in a dormant state (BOX 1).

\section{Setting the clinical stage}

Most of the work exploring tumour-directed PMN formation has used orthotopic and transgenic mouse models of metastasis, and most of our understanding of PMN biology is based on studies of lung metastasis, one of the most frequent sites of metastasis in both preclinical models and patients ${ }^{17,18}$. Preclinical models of metastasis enable researchers to test therapies in laboratory conditions that mimic disease present in patients. Nonetheless, there are several challenges associated with setting up spontaneous metastasis experiments such as the limited availability of syngeneic mouse models and the fact that metastasis is not confined to one metastatic organ ${ }^{18}$.

Currently, technical limitations, such as the lack of specific probes to track PMNs in real time by positron emission tomography (PET) and nuclear magnetic resonance (NMR) or the difficulty of obtaining premetastatic tissues from patients, remain the biggest obstacles in clarifying the clinical importance of PMNs for future metastasis. However, this gap in knowledge is now being addressed by several groups. These include a large cohort study assessing PMNs and metastatic niches in omental tissues from patients undergoing surgical resection of primary ovarian carcinomas (F. Balkwill, personal communication), as well as studies in premetastatic livers from patients undergoing surgical resection of primary pancreatic tumours (D.L. and W. R. Jarnagin, unpublished data).

Improving the understanding of PMN biology in patients requires the development of new technologies for better imaging techniques, such as the development of imaging probes to differentiate PMNs from healthy tissue. Thus, efforts to further uncover the clinical relevance and other ramifications of these niches are ongoing. For instance, much research is needed to explore the possibility of persisting PMNs that coexist once metastatic niches are created in a specific organ site and to determine whether established metastatic niches (or metastases on their own) can modulate secondary PMNs at additional organ sites of future metastasis (FIG. 1).

\section{Organ-specific PMNs}

Increasing clinical evidence reveals the existence of PMNs in tissue samples derived from cancer patients, as observed in sentinel lymph nodes (LNs) resected from patients with colorectal, prostate, breast, thyroid, bladder, gastric and renal cell carcinomas. ${ }^{8}$. LN PMNs were identified in mouse models of experimental metastasis and in patients with breast cancer, showing that provasculogenic vascular endothelial growth factor receptor 1 (VEGFR1)+ myeloid progenitor cells colonize LNs primed by tumour-secreted factors before the arrival of $\mathrm{CTCs}^{19}$. Subsequently, it was demonstrated that lymphangiogenesis precedes CTC arrival at future sites of LN metastasis ${ }^{20,21}$. Tumour cells encounter immune cells within LNs and the lung and interact with them directly or indirectly through tumour-secreted factors or $\mathrm{EV} \mathrm{s}^{22,23}$. These interactions likely modulate immune responses against $\mathrm{CTCs}^{24}$.

Liver PMN formation, which occurs during the initial phases of visceral metastasis (that is, metastasis of gastrointestinal tract cancers, breast cancer and melanoma), also relies on BMDC recruitment ${ }^{25-27}$. Enzymes involved in ECM remodelling, such as tissue inhibitor of metallopeptidase 1 (TIMP1), generate liver PMNs through stromal cell-derived factor 1 (SDF1; also known as CXCL12)-C-X-C chemokine receptor type 4 (CXCR4)-dependent recruitment of neutrophil ${ }^{28}$. More recently, exosomes were demonstrated to be biomarkers and functional contributors to liver $\mathrm{PMNs}^{29}$. Specifically, exosomes derived from human pancreatic cancer cell lines expressing macrophage migration inhibitory factor (MIF) 'educated' Kupffer cells to produce transforming growth factor- $\beta$ (TGF $\beta$ ), which promoted ECM remodelling by hepatic stellate cells, enabling recruitment of bone marrow-derived macrophages. Thus, exosomal MIF levels may serve as an early biomarker for liver PMN 
Extracellular matrix

(ECM). Comprising molecules, specifically proteoglycans and fibrous proteins (fibronectin, collagen, elastin and laminin) secreted by stromal cells into the microenvironment, that generate an intricate network of macromolecules that fill the intercellular space.

Orthotopic

Derived from the Greek orthos, meaning right and topos,

meaning place, this terminology refers to grafting a tumour into the place in the body where it would normally arise and grow.

\section{Transgenic}

Relating to or denoting an organism that contains genetic material into which DNA from an unrelated organism has been artificially introduced.

Omental tissues

A double fold of peritoneum attached to the stomach and connecting it with certain organs of the abdominal viscera, composed of the greater and the lesser omentum, which are the membranes of the bowels.

\section{Neutrophils}

Also known as polymorphonuclear leukocytes. Mature granular white blood cells with a multilobular nucleus and cytoplasm containing very fine granules. They are typically the first responders to acute inflammation, such as bacteria infection, injury or certain cancers.

Exosomes

Extracellular vesicles (typically $30-150 \mathrm{~nm}$ in diameter) of endocytic origin, released into the extracellular space by all cell types through the fusion of multivesicular bodies with the plasma membrane.

\section{Kupffer cells}

Specialized liver-resident phagocytic macrophages that line the walls of the liver sinusoid blood vessels.

Stellate cells

Pericytes that reside in the area between liver sinusoid blood vessels and hepatocytes They play a prominent role in liver fibrosis and may function as liver-resident

antigen-presenting cells. a Single-organ PMN formation with monoclonal metastasis

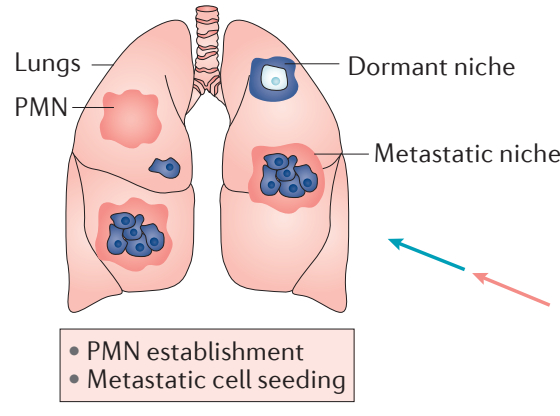

b Multiple-organ PMN formation with organ-specific monoclonal metastasis - Metastatic cell seeding
C Multiple and organ-specific PMN formation with monoclonal or polyclonal metastasis

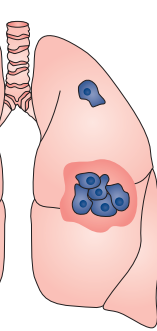

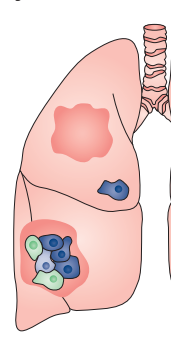
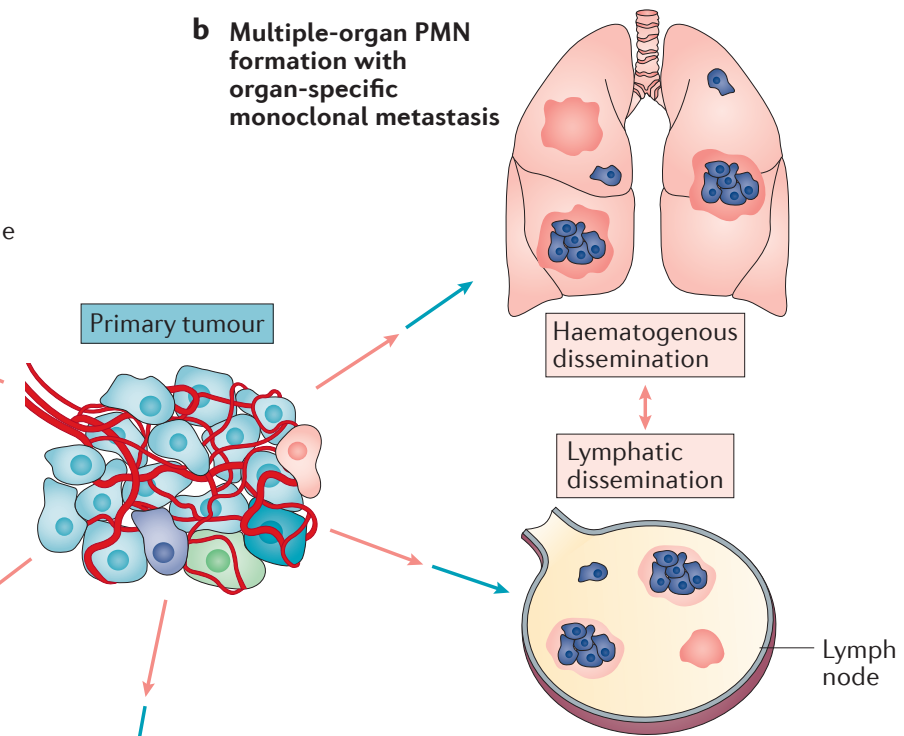

Figure 1 | Factors involved in PMN formation. a | Tumour-secreted factors and extracellular vesicles (EVs) shed by the primary tumour (blue arrows) induce pre-metastatic niche (PMN) formation in target organs before metastatic cell seeding occurs. Tumour cells seeding (pink arrows) the PMN thrive and give rise to micrometastasis. Cells seeding non-PMN areas lack a supportive tissue microenvironment and fail metastatic colonization. Specific niches — known as dormant niches - have contrasting effects to PMNs on circulating tumour cells (CTCs), promoting tumour cell dormancy instead of metastatic outgrowth. Molecules such as thrombospondin 1 (TSP1), deposited around stable microvasculature in response to bone morphogenetic protein (BMP) and transforming growth factor- $\beta$ (TGF $\beta$ ) secreted by stromal cells, are crucial mediators of cancer cell quiescence and thus dormancy. Dormant niches may arise in specific metastatic organs that constitute a growth-suppressive microenvironment for disseminated tumour cells (DTCs) (see also BOX 1). $\mathbf{b} \mid$ In most types of solid cancer, a primary tumour has the ability to seed more than one organ. Tumour-secreted factors and EVs can promote PMN formation in different organs, modulating the fate of tumour cell seeding at each metastatic site. Organ-specific seeding has also been observed and extensively reported in the literature. Most often, organ-specific metastasis is the result of metastatic colonization by a specific tumour subclone. Metastatic dissemination can be achieved via the haematogenous or lymphatic system. Interestingly, some cancer types, such as sarcomas, preferentially spread through blood vessels ${ }^{200,201}$, whereas others, such as epithelium-derived cancers, spread via the lymphatic vessels ${ }^{200,201}$. $\mathbf{c}$ Distinct PMNs form in different organs as a result of seeding by specific subclones derived from the primary tumour or owing to distinct microenvironments present at secondary sites of metastasis. Organ-specific PMNs may promote the formation of polyclonal metastatic lesions, composed of different tumour cell subclones, as opposed to the 'traditional' view that metastatic lesions are monoclonal in origin.

formation. Another exosomal biomarker, glypican 1, may also function in liver PMN formation, as well as being an indicator of early pancreatic cancer ${ }^{30}$. Thus, the mechanisms of PMN formation at sites of visceral metastasis share common features, such as BMDC influx and the horizontal transfer of information via tumour-secreted factors and exosomes.

Bone is a common site of metastasis for many types of solid tumour ${ }^{31}$. Primary tumours and the circulating factors derived from them can condition target cells residing in the bone to support PMNs and metastatic cell colonization $^{14,32,33}$. The reciprocal crosstalk between tumour cells and bone cells creates an environment ripe for osteoblastic (that is, bone-forming) or osteolytic (that is, bonedegrading) metastases. In osteolytic metastases (for example, from breast cancers), hypoxic tumour-secreted factors, such as the ECM-modifying enzyme lysyl oxidase (LOX), are essential for the formation of PMN osteolytic lesions ${ }^{33}$ (discussed further below). Other secreted factors such as parathyroid hormone-related protein (PTHrP) $)^{34}$, interleukin-6 (IL-6) ${ }^{34-37}$ and matrix metalloproteinases (MMPs) ${ }^{38-40}$ are known to activate osteoblasts, releasing high levels of receptor activator of nuclear factor- $\kappa B(N F-\kappa B)$ ligand (RANKL), which in turn induces osteoclast differentiation. These factors in turn promote the survival, growth and continued pro-osteolytic signalling of cancer cells ${ }^{14,41}$. In osteoblastic metastases (for example, from prostate cancers) there is still scarce information regarding PMN formation. Primary tumour-secreted factors such as WNT, 
bone morphogenetic proteins (BMPs), fibroblast growth factor (FGF), insulin-like growth factor I (IGF1) and IGF2, endothelin 1, prostate-specific antigen (PSA; also known as KLK3) and vascular endothelial growth factor A (VEGFA) influence osteoblast activity directly or indirectly ${ }^{42-45}$ and therefore may be candidates for driving osteoblastic PMNs. Whether these factors could execute these changes distally is still a matter of study.

Last but not least, the brain, as an immunoprivileged site protected by the unique properties of the blood-brain barrier (BBB), remains a challenge, as brain metastasis is one of the deadliest complications of treatment failure $^{46}$. Several recent studies have provided considerable insight into the evolution of BBB vascular permeability during cancer metastasis and its consequences for therapeutic efficacy ${ }^{46-49}$. For example, reduction in expression of the astrocytic basement membrane component laminin- $\alpha 2$ and the $\mathrm{CD} 13^{+}$pericyte subpopulation are observed when BBB permeability is compromised ${ }^{47}$. Although the brain PMN remains mostly uncharted territory, glucose metabolism is one of the most relevant mechanisms involved in breast cancer metastasis to the brain $^{50}$ as will be discussed further below.

\section{Secreted factors in PMN formation}

The establishment of PMNs facilitates organotropic metastasis by promoting CTC survival and outgrowth. This was shown first by injecting a Lewis lung carcinoma (LLC) cell line, which metastasizes only within the lung, into the flank of syngeneic mice pretreated with melanoma-conditioned medium (MCM). In MCMtreated mice, LLC cells now metastasized to spleen, kidney, oviduct and intestine, which are organs associated with melanoma metastasis ${ }^{7}$. This demonstrated that tumour-derived factors were a crucial component of MCM, and it was later shown that tumour-secreted exosomes exert crucial effects on PMN establishment (FICS 1,2). Indeed, tumour-derived exosomes dictate organotropic behaviour ${ }^{51}$, raising the question of what characteristics distinguish PMNs established in each of these organs. These studies reinforce the importance of the PMN in metastasis development by showing that tumour cell-intrinsic metastatic propensities are not sufficient for metastatic seeding and outgrowth and that a preconditioned microenvironment is the key determinant of organ-specific metastasis (TABLE 1).

\section{The pro-metastatic secretome}

Pro-angiogenic factors, such as VEGFA and placenta growth factor (PLGF) ${ }^{7}$, as well as inflammatory cytokines and chemokines, such as TGF $\beta$ and tumour necrosis factor (TNF), released by melanoma (B16 cells) and lung cancer (LLC cells) primary tumour cells were the first molecules shown to support PMN formation in the lung ${ }^{52,53}$ (TABLE 1). Furthermore, of the factors supporting BMDC recruitment to future PMNs, tumour-secreted granulocyte colony-stimulating factor (G-CSF) is sufficient to mimic the pre-metastatic lung microenvironment initiated by primary tumours and to enhance metastatic properties in both metastatic and non-metastatic mouse syngeneic tumours ${ }^{54}$.
Another tumour-derived chemokine with a crucial role in PMN lung formation is chemokine $\mathrm{C}-\mathrm{C}$ motif ligand 2 (CCL2). CCL2 is a potent chemoattractant for monocytes, macrophages, memory $\mathrm{T}$ lymphocytes and natural killer (NK) cells ${ }^{55}$. The important functions of CCL2 in cancer progression are reflected by the correlation between its overexpression and poor prognosis in many types of primary tumour ${ }^{55-60}$. Primary breast tumour cell-derived and stromal cell-derived CCL2 can also modulate the earliest steps of metastatic colonization following PMN formation by recruiting inflammatory monocytes that facilitate CTC extravasation in a VEGFA-dependent manner, thus effectively promoting lung micrometastasis ${ }^{61}$. CCL2 also inhibits NK cell maturation in the lung PMN in experimental models of both breast cancer and melanoma, probably decreasing the ability of NK cells to eliminate incoming $\mathrm{CTCs}^{62}$. Moreover, in MDA-MB-231 breast cancer cell xenograft models, CCL2 promoted breast cancer metastasis to lung by recruitment of mature macrophages to promote invasion and early establishment of tumour cell clusters, and to bone by enhancing osteoclast differentiation and enhancing the 'vicious' cycle of bone metastasis ${ }^{63,64}$. By contrast, in the $4 \mathrm{~T} 1$ syngeneic model of breast cancer, CCL2 induced neutrophil-mediated cytotoxicity in the premetastatic lung via elevated G-CSF levels, therefore inhibiting tumour cell seeding in PMNs, as evidenced by the reduction in number, but not size, of metastatic foci ${ }^{65}$. Collectively, these findings suggest multifaceted roles of CCL2 in PMN evolution and the establishment of early metastatic niches.

A common denominator of inflammatory responses within PMNs is the S100 family of proteins ${ }^{66}$. S100 proteins are inflammatory mediators that are crucial in intercellular crosstalk between tumour cells and stromal cells during PMN formation ${ }^{67}$. One important characteristic of these proteins is that they act both intracellularly and extracellularly. Within cells, S100 proteins regulate proliferation, differentiation, apoptosis, $\mathrm{Ca}^{2+}$ homeostasis, migration and invasion through interactions with cytoskeletal proteins, enzymes, transcription factors and nucleic acids ${ }^{68}$. Extracellular S100 proteins have both autocrine and paracrine effects via activation of cell surface receptors, G-protein-coupled receptors, scavenger receptors, heparan sulfate proteoglycans and $\mathrm{N}$-glycans ${ }^{67,68}$. They are involved in processes such as enhancement of $\mathrm{Ca}^{2+}$ influx in cells, cytokine production, immune cell recruitment and infiltration as well as inflammation ${ }^{68}$. Tumour-secreted factors, such as TNF, TGF $\beta$ and VEGFA, induce S100A8 and S100A9 expression by endothelial cells and by $\mathrm{CD}_{11} \mathrm{~b}^{+}$myeloid cells in the pre-metastatic lungs ${ }^{52,53,69}$. During this process, in mouse models, S100 proteins promote the expression and secretion of serum amyloid A3 (SAA3), which in turn recruits myeloid cells and tumour cells to $\mathrm{PMNs}^{53}$. This process requires SAA3-mediated activation of Toll-like receptor 4 (TLR4) and NF- $\kappa \mathrm{B}$ signalling ${ }^{53}$. Interestingly, as demonstrated in preclinical models of breast cancer, activation of hypoxia-inducible factor 1 (HIF1) is crucial even in PMN formation ${ }^{70,71}$. Indeed, 
the HIF1 target LOX is one of the main proteins induced at PMNs ${ }^{33,72,73}$. HIF1 $\alpha$-mediated hypoxia responses within the primary tumour may reinforce the secretion of both protein factors and EVs that are important in PMN formation and metastatic potential ${ }^{74}$. For example, in breast cancer cell lines, EV shedding is increased by the HIF-dependent expression of the small GTPase $\mathrm{RAB} 22 \mathrm{~A}^{75}$. It remains to be determined whether hypoxia or HIF signalling alters tumour-derived vesicle cargo and subsequently the education of stromal cells in distant PMNs.

\section{Tumour-derived messengers: EVs}

EVs are classified on the basis of their size and origin as microvesicles $(>150 \mathrm{~nm})$, which are formed at the cell surface through a budding mechanism, and exosomes $(30-150 \mathrm{~nm})$, which are produced in the endolysosomal and multivesicular body compartments for release outside the cell ${ }^{76,77}$. The machineries involved in the release of EVs, their docking and fusion during both physiological and pathological processes are still being deciphered. EV movement within the cell requires the cytoskeleton (actin and microtubules), associated molecular motors

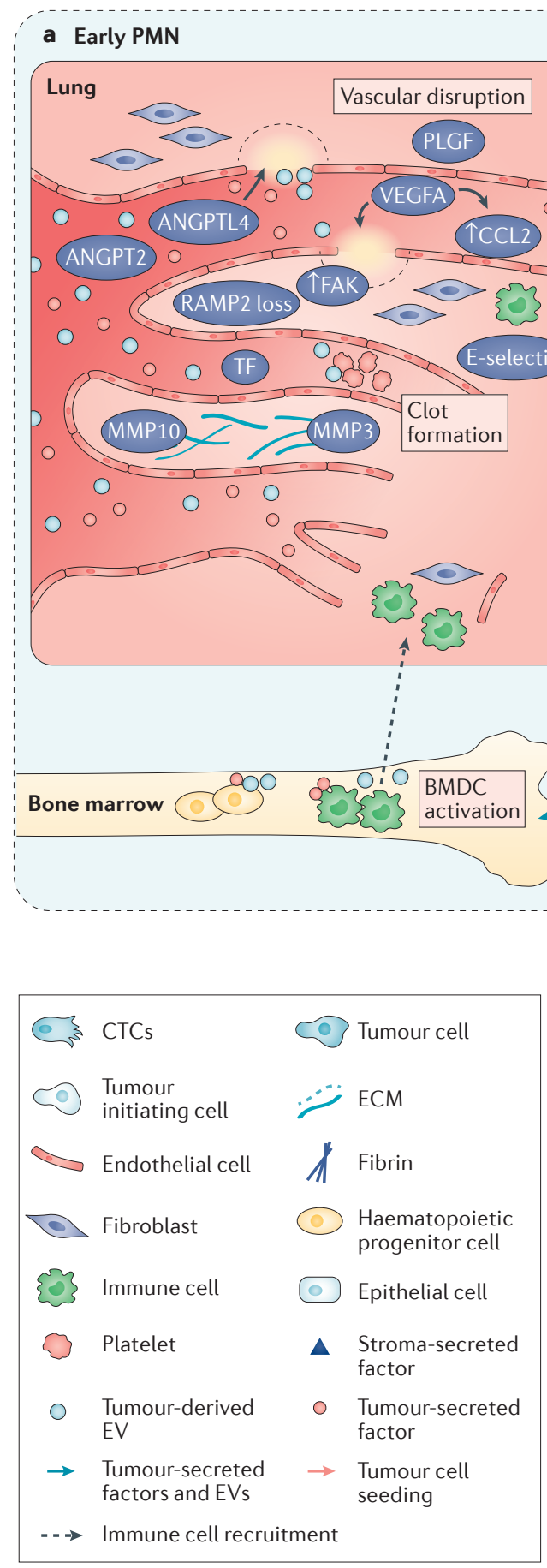

b Evolving PMN

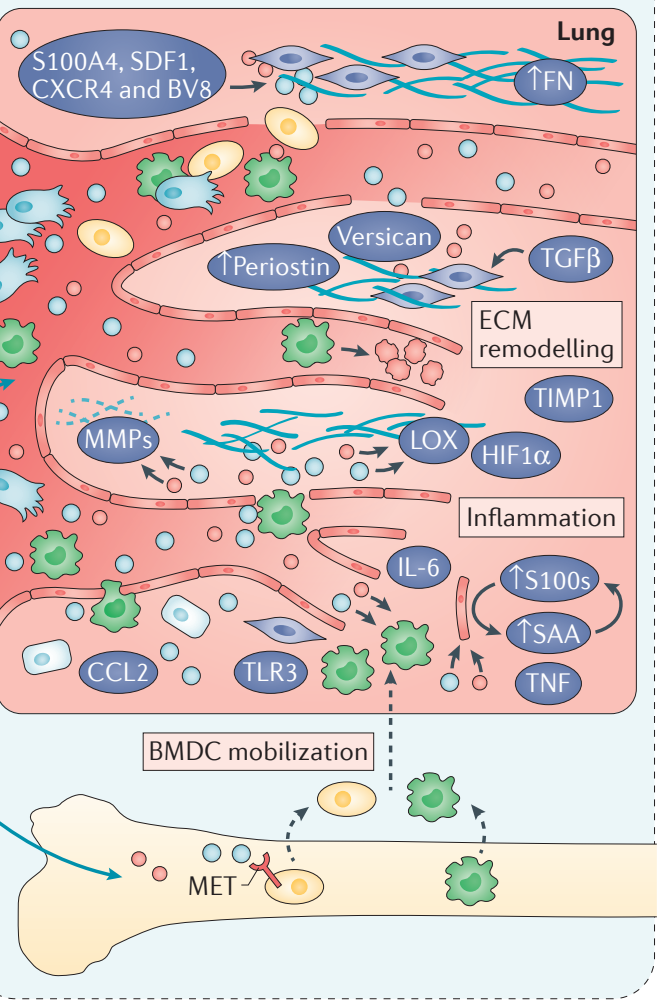

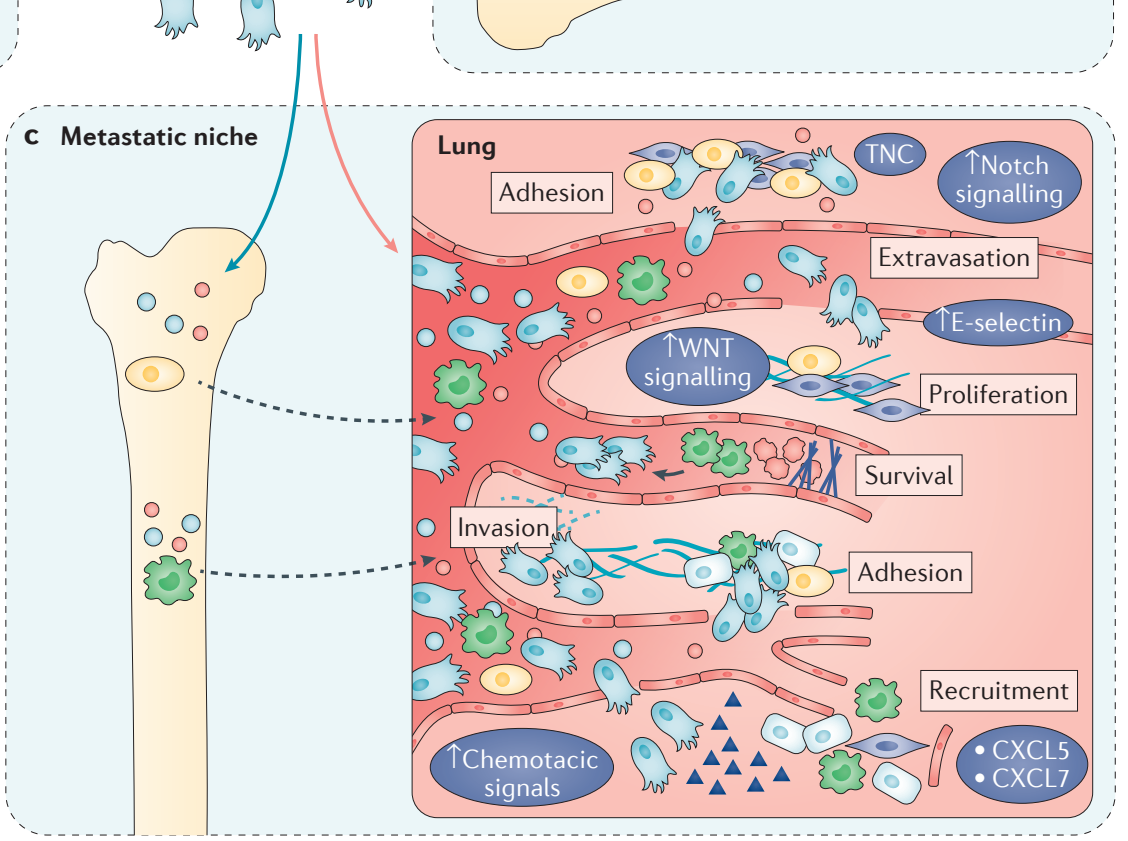


Cancer stem cells

A subset of cancer cells that share features of normal stem cells, such as self-renewal and differentiation and that can regenerate the tumour. (kinesins and myosins), molecular switches (small GTPases) and the fusion machinery (SNAREs and tethering factors $)^{77,78}$. Metastatic tumour cells demonstrate increased ability to package and secrete cargo (that is, proteins and microRNAs (miRNAs)) in exosomes ${ }^{29,79,80}$. Similarly, some stimuli that act during PMN formation, such as hypoxia, promote the release of exosomes ${ }^{74}$. Technical limitations in analysing PMNs (that is, requirement for in vivo microscopy) have hindered the analysis of EV dynamics within PMNs. Tumour-derived EVs can be isolated from bodily fluids such as plasma and carry tumour cell-derived proteins and genetic material that promote disease progression ${ }^{77,81}$.

Tumour cell-derived EVs fuse with resident cells in both PMNs and tumours, transferring their cargo, including genetic material (DNA, mRNA and miRNA), metabolites (lipids and small metabolites) and proteins ${ }^{79,82-86}$. Pioneering studies demonstrated that platelet-derived EVs (particularly microparticles) induced

\footnotetext{
4 Figure 2 | Model of PMN formation. a Establishment of the pre-metastatic niche (PMN). Clot formation and vascular disruption are hallmarks of the initial step of PMN formation. Tumour-secreted factors such as vascular endothelial growth factor A (VEGFA) and angiopoietin-like 4 (ANGPTL4) enhance the permeability of microvessels in the lung, resulting in loss of vascular integrity and enhanced metastasis. The mechanisms underlying such alterations are usually associated with disruption of endothelial cell-cell contacts through focal adhesion kinase (FAK) activation. Additionally, local increase in cytokines such as chemokine C-C motif ligand 2 (CCL2), of both tumour and stromal origin, correlates with the recruitment of various bone marrow-derived cell (BMDC) populations, which aid cancer cell extravasation and further contribute to PMN formation. Clotting factor production by platelets and accumulation of platelets in pre-metastatic organs, through deposition of proteins such as tissue factor (TF), promote the initiation of coagulation and clot formation. Clots lead to reduced shear stress and reduced interstitial flow, thereby providing docking sites for the arrest of circulating tumour cells (CTCs) and are a source of inflammatory signals ${ }^{108,112}$. In parallel, tumour-secreted factors influence the bone marrow niche, where BMDC populations become activated and primed for their future recruitment to the PMNs. Extracellular vesicles (EVs) have recently been suggested as having a crucial
} role in the establishment of PMNs. b| Evolution of the PMN. During PMN formation, extracellular matrix (ECM) is actively deposited and remodelled. Accumulation of fibronectin (FN) and crosslinking of collagen I (via lysyl oxidase (LOX)) provide a platform for the adhesion of BMDCs. Distant and local secretion of matrix metalloproteinases (MMPs), which also contributes to the early vascular disruption steps, promotes ECM degradation and aids future CTC invasion. Adhesion molecules present on tumour-derived exosomes, such as integrins, can bind to ECM molecules in organ-specific PMN niches, thereby determining organotropic metastasis. Transforming growth factor- $\beta$ (TGF $\beta$ )-dependent production of proteins such as periostin, also has an important role in the establishment of the PMN by sustaining the future infiltration of metastasis-initiating cells via WNT signalling. Upregulation of S100 proteins (particularly S100A8 and S100A9) generates inflammation and leads to the subsequent recruitment of haematopoietic progenitor cells as well as differentiated innate and adaptive immune cells (monocytes, macrophages and myeloid-derived suppressor cells (MDSCs), T cells, T regulatory cells and natural killer (NK) cells). c| Metastatic niche formation. The established metastatic niche is the combined result of BMDC recruitment, underlying ECM changes and inflammation. For instance, changes in vascular ECM composition or in the expression of endothelial molecules, such as E-selectin enrichment on the luminal surface of endothelium, facilitate CTC adhesion to the endothelium, extravasation and metastatic outgrowth. In addition, survival and proliferation of cancer cells in the metastatic niche also occur as a consequence of PMN evolution, which results from the complex action of various tumour-secreted factors and EVs on future tumour-seeding organs. ANGPT2, angiopoietin 2; CCL2, C-C motif ligand 2; CXCL, C-X-C motif chemokine ligand; CXCR4, C-X-C chemokine receptor type 4; HIF1 $\alpha$, hypoxia-inducible factor 1a; IL-6, interleukin-6; PLGF, placenta growth factor; RAMP2, receptor activity-modifying protein 2 ; SAA, serum amyloid A; SDF1, stromal cell-derived factor 1; TIMP1, tissue inhibitor of metallopeptidase 1; TLR3, Toll-like receptor 3; TNC, tenascin C; TNF, tumour necrosis factor. angiogenesis and metastasis in lung and breast cancers $^{87,88}$; however, the relevance of EVs in PMN formation was not evaluated in these studies. Since then, EVs have been shown to contribute to the recruitment and transfer of material to other stromal cell types, including those populating $\mathrm{PMNs}^{81,89-91}$.

Tumour-derived exosomal miRNA and proteins reprogramme or educate target cells that take up exosomes towards a pro-metastatic and proinflammatory phenotype, creating the $\mathrm{PMN}^{29,79}$. Education of stromal cells depends on the cancer type and pre-metastatic organ analysed. In melanoma, the hepatocyte growth factor receptor, MET, which is secreted in B16-F10 melanoma-derived exosomes, reinforces the expression of tyrosine kinase with immunoglobulin (Ig) and epidermal growth factor (EGF) homology domains 2 (TIE2) and MET in bloodcirculating BMDCs, and promotes pro-vasculogenic and pro-migratory properties in these cells. These reeducated BMDCs then exit the bone marrow and contribute to the formation of PMNs in lungs ${ }^{79}$. Importantly, exosomes secreted by B16-F10 melanoma cells promote vascular leakiness in lung PMNs, inducing a pro-inflammatory response by increasing the expression of cytokines and chemokines such as TNF, S100A8 and S100A9, which, in turn, recruit BMDCs to these $\mathrm{PMNs}^{79}$. However, the source of $\mathrm{S} 100$ proteins was not defined in this study. Breast and pancreatic cancer cell-secreted exosomes express integrins on their surface, which promotes their homing to $\mathrm{PMNs}^{51}$. Expression of the integrin $\alpha_{6} \beta_{4}$ heterodimer on the surface of tumourderived exosomes promotes their homing to lung PMNs, whereas $\alpha_{v} \beta_{5}$ targets them to liver PMNs. The homing of exosomes to these organs caused an increase in proinflammatory $\mathrm{S} 100$ family proteins in the PMN, generating a supportive microenvironment for subsequent metastasis ${ }^{51}$. Interestingly, human renal cancer stem cells promote angiogenesis and the formation of PMNs in the lungs, which involves $\mathrm{CD} 105^{+}$microvesicles inducing the expression of pro-angiogenic mRNAs and miRNAs in the whole organ ${ }^{92}$.

LN PMNs can be induced by intra-footpad injection of B16-F10 melanoma-derived exosomes ${ }^{93}$. In this study, genes associated with angiogenesis and ECM modification were highly upregulated in LNs; however, the cell types involved in the secretion of these factors were not identified. CD44 variant isoform (CD44v) secreted by pancreatic cancer cells enables the formation of specific microenvironments that favour the homing of tumoursecreted exosomes, inducing PMN formation in lung and $\mathrm{LN}^{22}$. Regarding liver PMNs, pancreatic tumour-derived exosomes expressing MIF promote TGF $\beta$ secretion in Kupffer cells, stimulating hepatic stellate cells to secrete fibronectin and promoting the recruitment of BMDCs ${ }^{29}$.

Interestingly, genomic content (that is, miRNAs) is also packaged selectively within EVs and is involved in PMN formation ${ }^{50,82,84,94}$. Tumour-derived microvesicles can suppress glucose uptake by stromal cells in brain PMNs through the transfer of miR-122 and inhibition of pyruvate kinase. This increases glucose availability in PMNs, attracting tumour cells, thus favouring brain metastasis ${ }^{50}$. 
Recently, RNAs packaged within primary tumour-derived exosomes were found to be involved in the activation of TLR3-dependent signalling in lung epithelial cells, inducing chemokine secretion in the lung and promoting PMN formation by recruiting neutrophils ${ }^{95}$.

These findings are only the beginning of our understanding of how tumour-derived EVs are involved in metastasis. There are many outstanding issues awaiting further research. First, it is imperative to determine which subpopulation of tumour cells gives rise to EVs that induce PMN formation. Second, a full characterization of secreted vesicles and their associated cargo is needed to understand how metabolic alterations affect PMN formation. Third, how long does EV-mediated

\section{Box 1 | The 'sleepy niche': tumour cell dormancy}

The biology of certain tumours demonstrates that, instead of forming pre-metastatic niches (PMNs) that increase the efficiency of metastasis, specialized microenvironments in which tumour cells could survive in a dormant state may occur. One could consider these niches as silent PMNs, which could result in extensive delay to the development of metastasis, as occurs in some patients. Defining the biology of both active and silent PMNs could help in understanding the different processes of metastasis. The enduring message of Paget's 1889 publication in the Lancet ${ }^{1}$ is his now famous 'seed and soil' hypothesis, which explains why breast cancer cells exhibit a proclivity for metastasizing to certain organ sites. Largely ignored was the contemporary view of Julius Cohnheim, of whom Paget said: “... [he was] of [the] opinion that a healthy organ has a certain ability to resist the growth of such an embolus; and he speaks of 'diminished resistance' as Fuchs speaks of "predisposition'." (REF. 5). How do healthy tissues resist the outgrowth of disseminated tumour cells (DTCs)?

DTCs persist long term essentially in a single-cell state within organs that succumb to metastasis as well as in other organs that rarely do ${ }^{190-192}$. The cellular and molecular constituents of the niches that promote DTC dormancy are now being elucidated. Two themes have emerged. The first is that niche-based cues function only in the context of specific tissues ${ }^{175}$. Factors that induce and sustain dormancy of DTCs from breast cancer, prostate cancer and head and neck squamous cell carcinoma act exclusively in lung (for example, bone morphogenetic protein 4 (BMP4) ${ }^{193}$ ) or in bone marrow (BMP7 and transforming growth factor- $\beta 2$ (TGF $\beta 2)^{194,195}$ ) despite their presence in other tissues, suggesting that they act in concert with additional factors. Whereas thrombospondin 1 (TSP1; also known as THBS1) suppresses DTC outgrowth in both lung and bone marrow $^{196}$, neither TSP1, BMP4, BMP7 nor TGF $\beta 2$ affect DTC dormancy in brain ${ }^{196}$. Indeed, a mediator of DTC dormancy specific to brain — or one that induces quiescence systemically - has yet to be uncovered.

The second major theme is that maintenance of tissue homeostasis is crucial. Most dormant breast cancer DTCs associate with the abluminal surface of the microvasculature of distant organ sites ${ }^{196}$. Using organotypic models of microvasculature, stable endothelium was shown to drive DTC quiescence, and perivascular TSP1 was a major endothelium-derived suppressor of DTC outgrowth ${ }^{196}$. But, how are these suppressive cues overcome? When microvascular homeostasis is disrupted, not only do sprouting vessels fail to express appreciable levels of TSP1, but endothelial tip cells deposit many of the factors found in PMNs - fibronectin, TGF $\beta 1$, periostin, tenascin C, versican and $\mathrm{S} 100$ proteins ${ }^{196}$. Whereas endothelial quiescence can be disrupted by any number of processes (for example, inflammation, wounding and ageing), it is also likely that primary tumour-derived factors released into the circulation affect the perivascular niche ${ }^{197,198}$. Although the primary tumour can be removed by surgical resection and patients can typically experience metastatic relapse after decades, it is important to realize that tumour cells can disseminate very early during tumour progression ${ }^{199}$. Therefore, dissemination, induction of DTC quiescence and disruption of these controls may have already occurred by the time the primary tumour is detected; a nuance that is not considered in the clinical definition of dormancy, which is a clinically asymptomatic state in which residual DTCs remain at undetectable levels for 5-7 years after seemingly successful treatment of the primary tumour. Primary tumour-derived factors may be the source of this dormancy disruption, destroying the dormant niche and inducing a PMN by altering microvascular homeostasis of specific tissues. If true, this would essentially marry and validate both Paget's and Cohnheim's theories. education of recipient cells last? Can EV-mediated education be reverted? Can educated cells be further re-educated by exposure to new EV content? And, are these cells more or less sensitive to repeated EV exposure? Lastly, much emphasis is now placed on understanding how EVs form, adhere, fuse and educate recipient cells within PMNs, which is crucial to dissecting EV involvement at all stages of PMN formation.

\section{The establishment of PMNs}

The stepwise progression of PMN formation is initiated with local changes such as the induction of vascular leakiness, remodelling of stroma and ECM, followed by dramatic systemic effects on the immune system ${ }^{96,97}$ (FIG. 2).

\section{Local changes in the PMN}

Vessel barrier breakdown: sites of vascular leakiness and beyond. The vasculature at PMNs is remodelled by primary tumour-secreted factors through various mechanisms. Animal tumour models show increased vascular permeability at $\mathrm{PMNs}^{98,99}$, which is associated with enhanced metastatic burden ${ }^{79,98,100,101}$. Altered vascular integrity is manifested through hyperpermeability, aberrant morphology of vascular endothelium and breakdown of the vascular basement membrane in the pre-metastatic lungs of mouse models of melanoma ${ }^{98}$ and breast cancer $^{101}$. Secreted factors, such as the EGF receptor (EGFR) ligand epiregulin, cyclooxygenase 2 (COX2; also known as PTGS2), MMP1 and MMP2, can induce vascular permeability in primary breast tumours and distant organs, enabling CTCs to extravasate ${ }^{102}$. Genetic ablation of these secreted factors in breast cancer cells impaired their ability to metastasize when injected through the tail vein. These data suggest that secretion of these factors from primary tumours may also be involved in the disruption of vascular permeability at PMNs. Furthermore, TGF $\beta$ induces the expression of angiopoietin like 4 (ANGPTL4) in breast tumour cells, including CTCs, enhancing the permeability of microvessels in the lung ${ }^{103}$. In a melanoma model, primary tumour-secreted factors upregulated angiopoietin 2 (ANGPT2), MMP3 and MMP10 in the premetastatic lung, which synergistically destabilized blood vessels ${ }^{98}$. The specific identities of the cells expressing these molecules and the tumour-secreted factors that induce their expression have yet to be defined. Another member of the MMP family, MMP9, is intimately involved in regulating vascular integrity in $\mathrm{PMNs}^{7,52}$. Myeloid progenitor cells recruited to the pre-metastatic lung produce high levels of MMP9, leading to ECM remodelling and formation of a proliferative, immunosuppressive and inflamed PMN in the lung ${ }^{101}$. Genetic ablation of $M m p 9$ normalized the aberrant vasculature in the pre-metastatic lung, resulting in diminished lung metastasis and improvement of host immune surveillance ${ }^{101}$. These results suggest that MMPs expressed by tissue-resident cells at sites of PMN formation act in concert to destabilize the vasculature and create a PMN.

An active role for altered endothelial cells within the hyperpermeable area of PMNs has also been proposed ${ }^{98,100}$. Syngeneic breast and lung primary tumours 
Table 1 | Primary tumour determinants of organ-specific metastasis

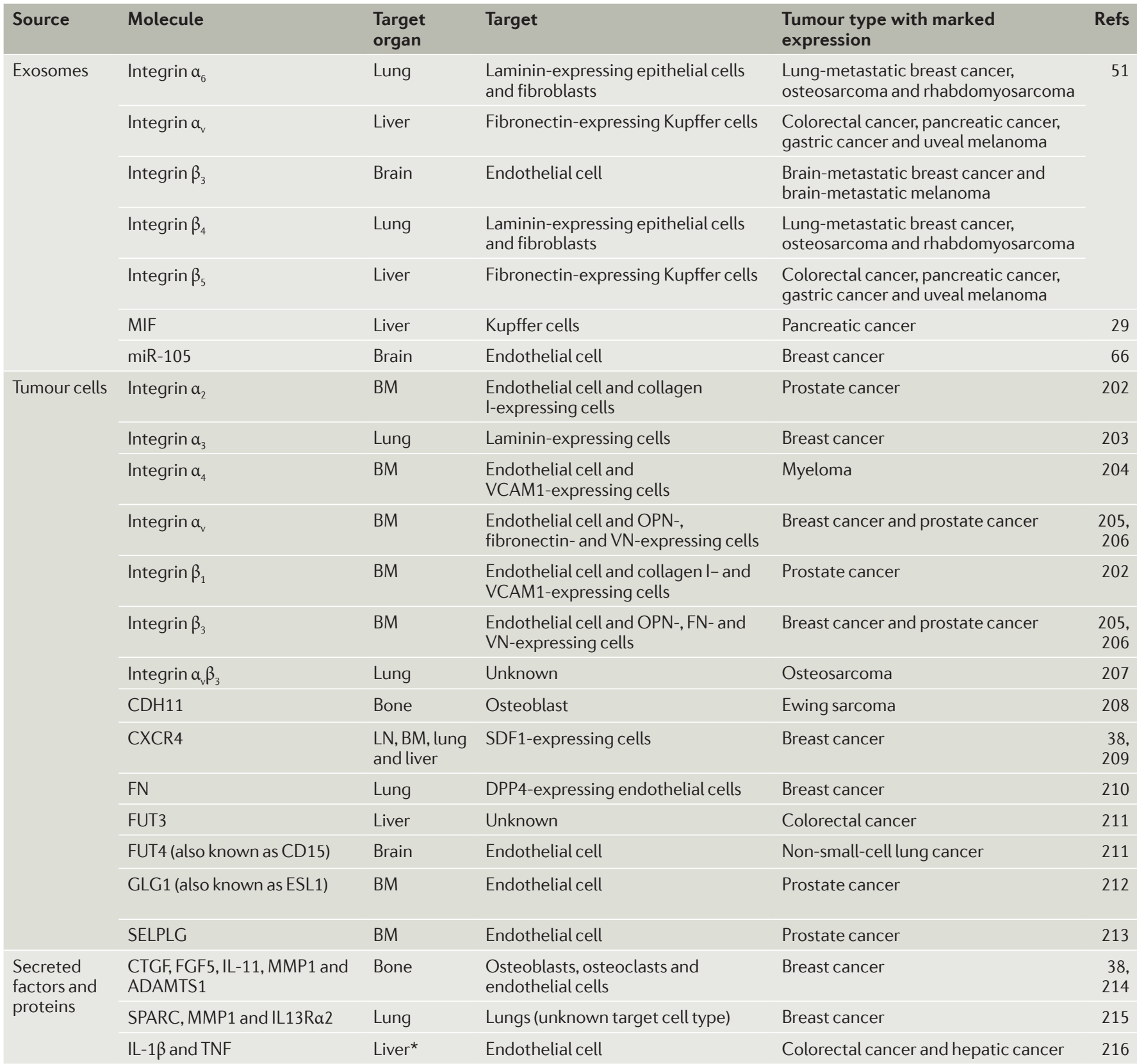

ADAMTS1, a disintegrin and metalloproteinase with thrombospondin motifs 1; BM, bone marrow; CDH11, cadherin 11; CTGF, connective tissue growth factor; CXCR4, C-X-C motif chemokine receptor 4; DPP4, dipeptidyl peptidase 4; FGF5, fibroblast growth factor 5; FN, fibronectin; FUT, fucosyltransferase; GLG1, Golgi apparatus protein 1; IL, interleukin; IL13Ra2, interleukin-13 receptor-a2; LN, lymph node; MIF, macrophage migration inhibitory factor; miR, microRNA; MMP1, matrix metalloproteinase 1; OPN, osteopontin; SDF1, stromal cell-derived factor 1; SELPLG, P-selectin glycoprotein ligand 1; SPARC, secreted acidic and rich in cysteine; TNF, tumour necrosis factor; VCAM1, vascular cell adhesion molecule 1; VN, vitronectin. *Not tested in vivo.

induce the formation of discrete foci within hyperpermeable vessels in the pre-metastatic lung. These foci were defined by E-selectin enrichment on the luminal surface of endothelium, which in turn facilitated CTC adhesion and outgrowth ${ }^{100}$. E-selectin upregulation is mediated by VEGFA-dependent induction of endothelial focal adhesion kinase (FAK) ${ }^{100}$ secreted by tumour cells or by injection of recombinant VEGFA, and inhibition of endothelial cell FAK hinders lung metastasis $^{104}$.
In breast cancer models, CCL2 secretion by tumour cells and the stroma leads to the recruitment of monocytes expressing C-C chemokine receptor type 2 (CCR2), that, in turn, secrete VEGFA ${ }^{61}$. Monocyte-specific depletion of VEGFA significantly inhibited breast cancer experimental metastasis potential and seeding efficiency ${ }^{61}$. Therefore, secreted factors could act synchronously by increasing both endothelial leakiness and the recruitment of BMDCs, which then promote PMN formation and hence metastatic colonization and outgrowt ${ }^{61,105}$. 
Fenestrated vasculature

A permeable type of vasculature that contains ultramicroscopic pores of variable sizes, usually found in kidneys and glands as well as in the circumventricular organs of the brain.

Venous thromboembolism (VTE). Refers to either of two blood clot-related conditions: deep vein thrombosis (DVT) or pulmonary embolism (PE). DVT occurs when a blood clot forms in a deep vein whereas a PE occurs when a blood clot breaks off and circulates to the lung.

Disseminated intravascular coagulation

Systemic activation of blood coagulation, leading to fibrin accumulation, which in turn results in the formation of microvascular thrombi in vital organs

T helper $1\left(T_{H} 1\right)$ cell Member of a subset of $\mathrm{CD} 4^{+}$ $T$ cells that can activate macrophages and mediate cellular immunity through secretion of interferon- $\gamma$ (IFN $\gamma)$, interleukin-2 (IL-2) and tumour necrosis factor- $\alpha$ (TNF $\alpha$ )
It is likely that metastatic organotropism requires specific interactions of tumour cells with endothelial cells in those organs ${ }^{102,106}$. Although these observations were not made in the context of PMN biology, transmigration of the $\mathrm{BBB}$ by cancer cells requires proteolytic processing of the junctional adhesion molecule B (JAMB; also known as JAM2) by cysteine cathepsin $\mathrm{S}^{107}$. By contrast, metastasis to organs with more fenestrated vasculature, such as the lung, liver or bone marrow, could depend almost exclusively on adaptation of tumour cells to specific microenvironments, as the microvasculature at these organ sites is comparatively permissive for tumour cell extravasation ${ }^{31,38}$.

A notable PMN-associated change at the vascular level is the formation of blood clots. Even though aberrant haemostasis has been associated with cancer progression and metastasis for more than a century ${ }^{108}$ and venous thromboembolism (VTE) is one of the leading causes of mortality in cancer patients ${ }^{109}$, the biological relevance of clots for cancer progression and the underlying mechanisms that regulate it are still largely unexplored. Intravascular fibrin clots that are rich in platelets surround metastatic tumour cells arrested in the vasculature, thus promoting tumour cell dissemination ${ }^{110}$. Tissue factor (TF)-mediated clot formation in lungs recruits populations of macrophages expressing CD11b, CD68, F4/80 and CX3C chemokine receptor 1 (CX3CR1) during PMN formation, thus enhancing CTC homing and survival ${ }^{111}$. Interestingly, platelets are required for the rapid recruitment of $\mathrm{CD} 11 \mathrm{~b}^{+} \mathrm{MMP9}^{+} \mathrm{Ly} 6 \mathrm{G}^{+}$granulocytes to tumour cells to form lung 'early metastatic niches'. This mechanism involves $\mathrm{C}-\mathrm{X}-\mathrm{C}$ motif chemokine ligand 5 (CXCL5) and CXCL7 secretion by platelets upon contact with different types of tumour cell (that is, breast cancer cells in a spontaneous mouse model of breast cancer), promoting tumour cell seeding and metastatic progression in the lungs ${ }^{112}$. Cancer complications such as disseminated intravascular coagulation, in which thrombosis and haemorrhage occur simultaneously, could then reinforce the secretion of factors that promote tumour metastasis. Further characterization of both processes in the context of PMN biology, as well as determining whether tumour-secreted and platelet-secreted EVs have a role in cancer-associated coagulopathies, may lead to the development of therapies that can reduce patient mortality.

Awakening the stromal residents. Perturbation of endothelial cell function is just one of the first alterations during PMN development, as other stromal cell types are activated by secreted factors from primary tumours and contribute to PMN establishment (FIG. 2). Fibroblasts remodel ECM at PMNs either by depositing new ECM components or by secreting enzymes that alter the existing ECM structure ${ }^{32}$ (discussed further below). S100A4 ${ }^{+}$ pulmonary fibroblasts are the main cell type incorporating exosomes derived from MDA-MB-4175 lung-tropic breast cancer cells ${ }^{51}$. Importantly, exosomal cargo induces the upregulation of specific S100 family members in stromal cells, depending on the tumour type ${ }^{51}$. Specifically, in vitro treatment of lung fibroblasts with exosomes derived from breast cancer cell lines upregulates S100A4, S100A6, S100A10, S100A11, S100A13 and S100A16, whereas the analogous treatment of Kupffer cells with exosomes derived from pancreatic cancer cell lines resulted in upregulation of S100P and S100A8 (REF. 51). Moreover, S100A8 and S100A9 are strongly induced in the lungs 24 hours after intravenous injection of exosomes derived from the highly malignant B16-F10 melanoma cell line ${ }^{79}$. Mice injected subcutaneously with LLC and 3LL lung cancer cell lines, then treated with monoclonal antibodies against S100A8 and S100A9, displayed fewer $\mathrm{CD}_{11 \mathrm{~b}^{+}}$myeloid cells in pre-metastatic lungs and dramatically reduced lung metastasis ${ }^{53}$. The induction of SAA1 and SAA3 by breast cancer-derived S100A4 or by intravenous injection of S100A4 resulted in the expression of pro-inflammatory molecules such as S100A8, S100A9, G-CSF and MMPs in an organ-specific manner ${ }^{113}$. In addition, SAA1 and SAA3 upregulation by S100A4 increased tumour cell adhesion to fibronectin (discussed further below) and BMDC recruitment to metastatic organs ${ }^{67}$. These observations suggest that S100A4 acts through mechanisms similar to S100A8 and S100A9 to regulate the formation of PMNs by promoting pro-inflammatory microenvironments both in the primary tumour and in $\mathrm{PMNs}^{114}$.

Additional stromal cells activated during PMN formation are tissue-resident macrophages. Indeed, liver Kupffer cells, upon stimulation by exosomes derived from pancreatic cancer cell lines carrying MIF, can induce fibronectin production by hepatic stellate cells and generate a fibrotic liver environment, which facilitates the recruitment of BMDCs to PMNs and thus PMN formation ${ }^{64}$. Pulmonary alveolar macrophages also participate in the lung PMN in a mouse model of breast cancer ${ }^{115}$. Tumour-preconditioned alveolar macrophages contribute to immunosuppression by inhibiting tumoricidal $T$ helper $1\left(T_{H} 1\right)$ cell function as well as reducing the number and hindering the maturation of tumour antigen-presenting dendritic cells in the pre-metastatic lung, thus supporting metastasis.

Remodelling the ECM. The ECM at pre-metastatic organ sites undergoes remarkable changes in response to systemic factors released from the primary tumour, activated stromal cells or recruited BMDCs ${ }^{15}$ (FIG. 2). Such changes can be achieved by two mechanisms, deposition of new ECM components and changing the physical properties of pre-existing ECM at PMNs.

Fibronectin deposition by activated stromal fibroblasts in the pre-metastatic lung or liver has been observed in mouse models of melanoma and pancreatic cancer, respectively ${ }^{7,64}$. Accumulation of fibronectin provides a permissive niche for the adhesion of BMDCs, which are crucial components of PMNs. In addition, tumour-derived factors, such as TGF $\beta$, induce the expression and secretion of periostin from stromal fibroblasts expressing $\alpha$-smooth muscle actin (aSMA; also known as ACTA2) and vimentin (VIM) in the pre-metastatic lung of the MMTV-PyMT mouse model of breast cancer ${ }^{116}$. Secretion and incorporation of periostin into ECM thus establishes a PMN that 
Metastasis-initiating cells

Rare tumour cells that have the capacity to survive and proliferate in distant metastatic sites.

Myeloid-derived suppressor cells

(MDSCs). A heterogeneous population of myeloid-derived immunosuppresive and pro-tumorigenic cells that suppress T cell function. They expand in number in pathological conditions and interact with other innate and adaptive immune cells to modulate their function. They universally express CD $11 \mathrm{~b}$, but can be further categorized in both mice and humans on the basis of expression of additional markers into granulocytic and monocytic lineages

Macrometastases The outgrowth of micrometastases that are histologically or radiologically detectable. sustains infiltrating metastasis-initiating cells by augmenting WNT signalling. First, periostin interacts with ECM molecules intracellularly and then it is deposited outside the cell ${ }^{117}$. So far, periostin has been described to interact with type I collagen, fibronectin, NOTCH1, tenascin $\mathrm{C}$ and BMP1. Through these interactions periostin engages integrins such as $\alpha_{v} \beta_{3}$ and $\alpha_{v} \beta_{5}$ and promotes cell motility by acting outside the cell ${ }^{117}$. In a mouse wound-healing model, periostin was upregulated in damaged tissue, forming a PMN-like microenvironment and promoting melanoma cell metastasis to wound sites ${ }^{118}$. More recently, periostin was found to be essential for the immunosuppresive function of myeloid-derived suppressor cells (MDSCs) in the pre-metastatic lung during breast cancer metastasis ${ }^{119}$.

Versican is an ECM proteoglycan with multifaceted roles in the PMN. In mouse models of LLC lung cancer injected via the tail vein, tumour-derived versican activated macrophages through TLR2 to generate an inflammatory microenvironment in the pre-metastatic lung ${ }^{105}$. By contrast, in MMTV-PyMT mice, the sources of versican are $\mathrm{CD} 11 \mathrm{~b}^{+}$Ly6 $\mathrm{C}^{\text {hi }}$ myeloid cells recruited to the pre-metastatic lung ${ }^{120}$. Knock-down of versican in BMDCs impaired lung metastasis in the MMTVPyMT model, underscoring the functional requirement for stroma-derived versican in PMN development ${ }^{120}$.

Enzymatic modulation of ECM structure at the PMN can affect vascular integrity (as discussed above) and generate biologically active ECM fragments ${ }^{72,121-124}$. Indeed, chemoattractant collagen IV peptides released by MMP2 activity in the pre-metastatic lung guide the homing of BMDCs and CTCs to PMNs and promote metastasis ${ }^{72}$. The major sources of these MMPs are BMDCs (for example, macrophages, CD $11 b^{+}$myeloid cells and VEGFR $1^{+}$ haematopoietic progenitor cells (HPCs)), stromal fibroblasts and endothelial cells $7,52,72,125,126$.

Another important class of ECM-shaping enzymes is the LOX family, which catalyses the crosslinking of ECM molecules; collagen is their primary substrate. Several studies have shown that collagen type I and type IV crosslinking by various members of the LOX family (including LOX, LOX-like 2 (LOXL2) and LOXL4) that accumulate in PMNs of various organs provides a platform for adhesion of $\mathrm{CD}_{11 \mathrm{~b}^{+}} \mathrm{BMDCs}^{72,70,127}$. Recruitment of CD $11 b^{+}$BMDCs subsequently contributes to PMN formation through a positive feedback loop of MMP secretion by CD11 b cells and further invasion and recruitment of BMDCs and CTCs owing to the elevated MMP activity ${ }^{72}$. Hypoxia, through HIF1 activity, is a crucial regulator of LOX expression in primary breast tumours ${ }^{72}$. In addition to tumour cells, activated fibroblasts have also been identified as a source of $\operatorname{LOX}^{72,70,32}$. However, to what extent collagen crosslinking by LOX is required for PMN formation in other types of cancer remains to be investigated.

It is noteworthy that one important consequence of ECM remodelling is the change in physical properties. Tension generated in the extracellular microenvironment can initiate a cascade of signal transduction events that act as a molecular switch in development, tissue homeostasis and disease progression, including metastasis ${ }^{128-132}$. For example, collagen crosslinking also results in increased tissue stiffness, which has been shown to directly support tumour cell seeding, survival and proliferation in vitro, and to enhance metastatic outgrowth in vivo ${ }^{32}$. Whether ECM remodelling at PMNs occurs through mechanical tension transduction and what the specific functional consequences of ECM remodelling at the PMN are, remain to be elucidated. Importantly, new approaches to studying PMN composition and function, such as in vivo implantation of ECM-coated poly ( $\varepsilon$-caprolactone) (PCL) scaffolds that mimic the ECM composition of the $\mathrm{PMN}^{133}$, will clarify the contribution of the ECM to PMN formation and evolution. Another crucial issue awaiting clarification is whether functionally distinct variants of ECM molecules resulting from alternative splicing (for example, fibronectin has at least 20 variants in humans ${ }^{134}$ ) fulfil distinct roles in ECM remodelling during PMN establishment, and if so, towards what functional outcome.

\section{Systemic changes: immune cell recruitment}

A hallmark of PMN initiation and evolution is the recruitment of BMDCs (FIG. 2). Indeed, the recruitment of HPCs expressing VEGFR1 and VLA4 (integrin $\alpha_{4} \beta_{1}$ ) to pre-metastatic organs fosters a supportive microenvironment for incoming $\mathrm{CTCs}^{7}$. Abrogation of these HPC clusters within pre-metastatic organs by either VEGFR1 antibody-mediated blockade or depletion of VEGFR $1^{+}$BMDCs reduced metastasis, indicating the essential role of these HPCs during PMN initiation? ${ }^{7}$. However, even though upregulation of inhibitor of differentiation 1 (ID1) gene expression is crucial for VEGFR $1^{+}$HPC mobilization during PMN formation ${ }^{135}$, the identities of the tumour-derived factors that induce ID1 expression and drive HPC mobilization have yet to be determined.

In addition to recruiting HPCs from the bone marrow, primary tumours also 'hijack' different types of immune cell, causing their abnormal differentiation and accumulation at PMNs. Notably, MDSCs impose immunosuppression at PMNs and promote metastasis ${ }^{28,64,101,136,137}$. MDSCs can either be recruited to PMNs or they may develop from tissue-resident myeloid populations. An ingenious intravital twophoton live imaging approach revealed that upon arrival in the lung, CTCs interact with distinct populations of resident phagocytes, and tumour cell cytoplasts are generated within the lung vasculature that are taken up by recruited BMDCs ${ }^{24}$. Next, tumour-ingesting myeloid cells collectively accumulate in the lung interstitium and promote the development of metastases ${ }^{24}$. MDSCs repress interferon- $\gamma$ (IFN $\gamma$ )-mediated immune responses while inducing the expression of proinflammatory cytokines, interleukins and SDF1 (REF. 101), further supporting the idea that generation of a proinflammatory, immunosuppressive PMN through recruitment of BMDCs is crucial for successful metastatic progression. Importantly, PMN-associated MDSCs also produce MMP9, a crucial regulator of ECM remodelling and the angiogenic switch that will later support the formation of micrometastases and macrometastases ${ }^{138,139}$. 
Type I interferon

A class II $\alpha$-helical cytokine essential for protection against viral infections that also plays important roles in bacterial infections, shock autoimmunity and cancer.

\section{Leukotrienes}

Products of the eicosanoid metabolism of leukocytes that mediate inflammation and allergic reactions.
The roles of neutrophils at PMNs are particularly intriguing. Neutrophils can be expanded, mobilized and recruited to PMNs via tumour cell-derived G-CSF, exosomes or via stromal cell-derived SDF1 in metastatic organs ${ }^{28,65,136,137}$. To date, the markers used to define neutrophils are oversimplified and thus identify a heterogeneous population marked by profound functional complexity. Further immunophenotyping is required to sort PMN-infiltrating immune cells for functional assessment. Nevertheless, recent data demonstrate that neutrophil recruitment to PMNs and their diminished cytotoxic activity both occur only in the absence of type I interferon, suggesting that interferon-based therapies could be beneficial in hindering PMN progression ${ }^{140}$. However, owing to the multifaceted role of IFN $\gamma$, the tumour-suppressive and tumour-promoting activities of which are normally dependent on the cellular, microenvironmental and/or molecular context, targeting it could be a double-edged sword and should be considered carefully ${ }^{141}$.

Neutrophils were shown to support the establishment of lung PMNs in mouse models of breast can$\operatorname{cer}^{142}$. Isolation of neutrophils derived from PMNs demonstrated that they secrete leukotrienes, converting heterogeneous cancer cell populations into metastasis-initiating cells and enhancing metastatic competence $^{142}$. Recently, lung epithelial cells have been described to initiate neutrophil recruitment and lung PMNs by sensing tumour exosomal RNAs through TLR3 (REF. 95). LLC-derived exosomal RNAs activate TLR3 in lung epithelial cells, inducing secretion of chemokines (CXCL1, CXCL2, CXCL5 and CXCL12) and promoting neutrophil recruitment. An interesting question stemming from these observations is: how do innate immune cells support PMN formation? Recent evidence supports the idea that, besides neutrophils, there could be active, EV-mediated communication between tumour cells and other innate immune cells during PMN formation ${ }^{143}$.

\section{Clinical implications of PMN}

Metastasis is the leading cause of cancer-associated death, yet its treatment has limited lasting success ${ }^{49}$. Thus, developing strategies to prevent metastasis, or to detect it at its earliest stages, is a priority for improving patient outcomes. Intriguing questions to consider are: can the PMN be manipulated to prevent seeding or outgrowth of incoming CTCs? Can non-metastatic tumours, their EVs or secreted factors exert systemic inhibitory effects on tissues to block metastasis?

\section{PMN detection}

Currently, patients with a newly diagnosed malignancy are routinely assessed for metastases by radiological imaging (for example, computerized tomography (CT) and positron emission tomography (PET)), by histology and cytology (for example, sentinel LN biopsies and bone marrow examination). Both PET and CT imaging ${ }^{144}$ are unreliable in detecting tumours less than $1 \mathrm{~cm}$ in size ${ }^{145}$, by which time the tumour cell burden is estimated at $10^{9}$ cells $^{146}$. Furthermore, PET-CT is frequently unable to differentiate between benign inflammatory and malignant lesions. And for practical reasons, neither routine use of biopsies nor assessment of all potential sites of metastasis is feasible ${ }^{147}$.

So how can the reliability of metastasis detection be improved? Much effort is now being invested in developing higher resolution imaging techniques and uncovering genetic and biochemical biomarkers of early metastasis. As such, imaging approaches that can enable detection of structural changes in the PMN, such as tissue den$\operatorname{sity}^{32,33}$, hold promise. Moreover, biochemical and cellular biomarkers of early metastasis in circulation, such as VEGFR1 expression by circulating BMDCs ${ }^{19,96,148-151}$ or $\mathrm{CD} 8^{+}$myeloid cells that have activated signal transducer and activator of transcription 3 (STAT3) ${ }^{152}$ have great potential as molecular tools for PMN detection and monitoring ${ }^{19,96,148-152}$. Importantly, sphingosine 1-phosphate receptor 1 (S1PR1)-STAT3 signalling activation in $\mathrm{CD}^{+} 8^{+}$myeloid cells is crucial for their recruitment to PMNs and future metastatic outgrowth ${ }^{153-155}$. Selective blocking of VEGFR1 using a novel peptide antagonist named iVR1 completely ablated liver PMN formation and liver metastasis of colorectal cancer cells in mice ${ }^{156}$. In addition to VEGFR1, other molecules, such as dickkopf 1 (DKK1), VEGFA, VEGFD and HIF1a could serve as PMN biomarkers in human LNs, predicting future metastasis in cancer patients ${ }^{157-159}$. Importantly, morphological and structural changes, such as fibronectin deposition and changes in collagen metabolism, occur in LN PMNs, resulting in changes in capsule thickness that could be used as a measurement of PMN development ${ }^{160}$. A fibrosis-like environment may precede tumour seeding and metastasis at PMNs in the liver ${ }^{29}$, supporting the need to image structural changes in tissue density possibly by biopsy of the liver PMN. Most importantly, clinical trials are ongoing to measure PMN formation, its diagnostic and prognostic power and whether blockade of PMN formation could be obtained by using, for example, pazopanib ${ }^{161}$, a multitargeted tyrosine kinase inhibitor against VEGFR1-3, platelet-derived growth factor receptor- $\alpha$ (PDGFRA), PDGFRB and KIT ${ }^{162}$.

EVs may be the most promising biomarkers for assessing risk of tumour progression and metastasis. Their accessibility, abundance and stability in the circulation make them ideal biomarker candidates. Developing techniques to enrich for tumour-derived EVs to define their selective cargo can improve the sensitivity of detection of tumour EV-associated biomarkers in blood from cancer patients $^{163,164}$. Alternatively, owing to the technological limitations (including obtaining fresh specimens and limited viability of tumour-derived explants) of detecting tumour-secreted factors that are involved in PMN formation, tumour organoids could be used to identify tumour-specific biomarkers that can be verified in patient plasma. Exosomal miR-105 derived from primary tumours from patients with breast cancer (the tumour cells of which expressed high levels of miR-105) could be detected in patient serum at an early stage before the clinical detection of metastasis ${ }^{165}$. Furthermore, exosomal levels of a melanoma-specific protein, tyrosinase-related protein 2 (TYRP2; also known as DCT), correlated with metastatic progression in a retrospective cohort of patients 
Dormancy

A latent state in which individual tumour cells are quiescent and reversibly arrested in $\mathrm{GO}$ phase of the cell cycle. with stage III melanoma (patients with LN metastasis) at the time the samples were taken, demonstrating that plasma exosomes can predict metastasis in melanoma ${ }^{79}$.

Proof-of-principle studies have demonstrated that loading of exosomes with superparamagnetic iron oxide nanoparticles or iodine isotopes enables sensitive magnetic resonance or radiographic tracking ${ }^{166}$. Ingenious approaches to noninvasive imaging of PMN formation in vivo, based on the role of VLA4 in PMN biology, combined the use of PET-CT with $\left[{ }^{64} \mathrm{Cu}\right] \mathrm{CBTE} 2 \mathrm{~A}-\mathrm{LLP} 2 \mathrm{~A}$ (a VLA4-targeted PET radiopharmaceutical) ${ }^{167,168}$ or relied on label-free quantitative detection methods of tumour-derived exosomes through surface plasmon resonance imaging ${ }^{169}$

If exosome-mediated reprogramming of systemic energy metabolism can facilitate disease progression, similar exosome-based strategies could be devised to hinder PMN formation. For example, exosomes can be engineered to deliver molecules that inhibit the repression of glucose uptake by stromal cells in brain PMNs. Therefore, there is hope for translating these advances to the clinic to exploit tumour exosomes as predictors of metastatic potential and organotropism, as well as therapeutic targets to block PMN formation and metastatic colonization.

\section{Preventing PMN formation and progression}

Targeted therapies directed against the establishment of PMNs could potentially stop metastasis in its tracks. For example, ATP release by breast cancer activates the P2Y purinoceptor 2(P2RY2) and subsequently the HIF1-LOX axis, which induces collagen crosslinking and the recruitment of CD11 $\mathrm{b}^{+}$BMDCs; inhibition of P2RY2 signalling proved an efficient strategy to prevent $\mathrm{PMN}$ formation in preclinical breast cancer models ${ }^{170}$. Similarly, downstream targeting of LOX by treatment with $\beta$-aminopropionitrile $(\beta \mathrm{APN})$, function-blocking antibodies or LOX-specific RNA interference showed promise in abolishing PMN formation in preclinical models of breast cancer ${ }^{33,72}$. In another approach, expression of protein tyrosine phosphatase receptor-type O (PTPRO, a multitarget negative regulator of VEGFA, PDGF and FGF receptor 1 (FGFR1)) in the mouse breast cancer cell line Py81119 prevented PMN formation in preclinical models of breast cancer, thus thwarting lung metastasis ${ }^{171}$. The CXCR4 inhibitor AMD3465 also obstructed myeloid cell recruitment to liver and lung PMNs, preventing metastatic progression in immunocompetent mouse models of breast cancer ${ }^{172}$. MET inhibition (either through small-molecule inhibitors or through humanized antibody blockade, both of which are approved by the US Food and Drug Administration (FDA)), could represent a two-pronged approach that would prevent the function of pro-metastatic melanoma exosomes as well as the recruitment of exosome-educated BMDCs to $\mathrm{PMNs}^{79}$. Importantly, substantial breakthroughs in understanding the biology of PMN formation, combined with advances in imaging and serum biomarker detection, could offer opportunities for timely targeting of PMNs and early metastasis as well as identifying patients who are likely to have aggressive disease.

\section{Future directions}

We have achieved tremendous progress in our understanding of the complex molecular mechanisms that govern PMN formation, but key questions remain unanswered.

\section{Can PMN progression be hindered?}

The redirection of experimental metastasis suggests that preconditioning of the microenvironment in specific organs, especially via exosomes, can increase their susceptibility to metastasis ${ }^{51}$. Therefore, an understanding of tumour exosome biogenesis, with the aim of selectively controlling tumour exosome production may prove a viable approach to reducing metastasis. Conversely, a better grasp on the biology of certain tumour-secreted factors with anti-metastatic functions may offer unique therapeutic opportunities. Can inhibitors of angiogenesis, such as angiostatin and endostatin ${ }^{173}$, be used to condition PMNs and thereby inhibit metastasis by normalizing the vasculature? Furthermore, are there microenvironments at sites of future metastasis that either do not require preconditioning by tumour-derived factors to support metastasis or are intrinsically inhibitory to metastatic outgrowth and thus capable of inducing dormancy of DTCs ${ }^{174,175}$ ?

\section{Are all PMNs created equal?}

Is PMN composition in different organs alike? Are PMNs in distinct organs induced by the same mechanisms? Moreover, what are the dynamics of PMN formation? Are PMNs in different organs created simultaneously or sequentially? Our observations suggest that the systemic conditioning of PMNs by various cancer types may differ ${ }^{29,51,79}$. Certain mechanisms, such as increased vascular permeability and recruitment of BMDCs, seem to be common for all tissues examined thus far, but specific molecules expressed and/or shed by the tumour (for example, chemokine receptors and integrins) could contribute to organotropic behaviour.

\section{Are there other components of the PMN?}

The PMN is a complex microenvironment, and less-explored resident and recruited cell types may fulfil crucial roles in PMN initiation and progression. For example, adipocytes contribute to metastasis by secreting adipokines and other cytokines (for example, leptin, adiponectin, osteopontin, TNF, IL-6, CCL5 and CCL2 (REFS 176-178)) and fatty acids to fuel rapid tumour growth at metastatic sites ${ }^{179}$, but their contribution to PMN formation remains unknown. Accumulating evidence indicates that activated sympathetic neurons can also induce a metastatic switch in mouse breast and ovarian cancer models by increasing tumour vascularization and invasion in the metastatic microenvironment via neuroendocrine signalling ${ }^{180}$. Sympathetic neuron signalling can also alter the cytokine profile of the bone microenvironment, which can induce osteolysis and promote the colonization of metastatic breast cancer cells in bones ${ }^{180}$, adding neurons to the tumour-stroma crosstalk within the PMN. 


\section{Can PMNs be biomarkers of metastasis?}

Early detection of PMNs before radiographic evidence of metastasis remains a challenge. New technologies that attempt to detect hyperpermeable regions, hypoxic and inflammatory areas, ECM alterations or BMDC recruitment at pre-metastatic stages are being developed ${ }^{166-168,181}$. More sensitive and translational technical approaches, such as labelling specific PMN probes (that is, by the use of inhibitors, antibodies or peptides recognizing the main proteins that are upregulated at PMNs) with radioisotopes, are needed to translate these findings to the clinical setting. Moreover, determining which PMN will transition to metastatic disease offers additional opportunities to improve diagnostic, biomarker and prevention potential associated with PMN detection.

\section{Why do secondary tumours develop?}

Secondary tumours (also known as metastasis) that arise after surgery are derived from the primary tumour cells. Given the evidence that primary tumours can precondition distant sites for metastasis, it is likely that such education by tumour-derived factors has already occurred and PMNs have already been formed before surgical removal of the primary tumour. Nevertheless, it is clear that surgery is followed not only by tumour cell shedding into the circulation, but also by a surge of inflammatory cells in the blood, thus favouring PMN formation ${ }^{182}$. Moreover, surgical intervention has been shown to induce local hypoxia and injury, which in themselves promote metastasis ${ }^{183-185}$, but the systemic implications for PMN formation have yet to be explored. Further studies will need to address whether tumour-secreted factors, such as EVs, are also increased after surgery and contribute to PMN development and thus support future metastasis, and whether non-steroidal anti-inflammatory drugs (NSAIDs), which reduce the most immediate and abundant peak of breast cancer recurrence (9-18 months after surgery) fivefold ${ }^{186}$ could function as a prophylactic that acts (at least partially) by reversing PMN formation in patients with breast cancer.

\section{Can the PMN be targeted?}

Does chemotherapy or radiotherapy promote PMN development? Specific targeting of PMN components (for example, inhibitors of VLA4, TNF, LOX or HIF) reduces metastasis in preclinical model ${ }^{7,33,53,73}$, but owing to current technical limitations in detecting PMNs and the lack of clinical trials to test these molecules in the premetastatic setting in patients, the efficacy of this approach in preventing metastasis in cancer patients has yet to be determined. One possible explanation for the inadequate control of cancer metastasis with current pharmacological approaches is the failure of drugs to reach the PMN owing to the increased permeability of the vasculature at PMNs (as discussed above). Vascular leakiness leads to an abnormal tumour microenvironment characterized by interstitial hypertension (elevated hydrostatic pressure outside the blood vessels), hypoxia and acidosis ${ }^{187}$. Stabilization of vasculature at PMNs could then help to improve specific strategies against PMN formation. Furthermore, the systemic consequences of any current or novel therapy on PMN formation are difficult to predict. For example, mobilization of haematopoietic stem and progenitor cells from their niches may alter the bone marrow microenvironment for malignant cells to engraft ${ }^{188}$. It is also possible that certain treatments may alter organotropism; for example, agents activating specific cell types such as osteoclasts may promote bone metastasis ${ }^{33}$.

\section{Summary}

In conclusion, remarkable progress in our knowledge regarding PMN formation and evolution has been made in the past decade since the PMN was first described. We envisage the PMN as a new paradigm for the initiation of metastasis and recognize that the pathological processes occurring before the development of macrometastases require better understanding. This is crucial, especially as in patients PMNs may not only be established in distant organs but may also develop in the vicinity of macrometastatic lesions in the same organ. Moreover, treatment of macrometastasis (for example, by targeting angiogenesis), can promote micrometastasis ${ }^{189}$ and could also promote PMN formation, warranting the identification of therapies that could thwart all processes simultaneously. The primary tumour-directed induction of the PMN enforced by tumour-secreted factors can dictate metastatic burden and distribution, and the cellular make-up of future PMNs is an area ripe for exploration. The study of PMNs could benefit from 3D modelling of organ-specific niches, to enable a systematic dissection of the cellular and ECM components of the PMN. The challenge that lies ahead, to bring the advances in our understanding of PMN biology to the bench, is translation of this complex biology to diagnostic and treatment approaches. This will require innovative, well-designed clinical trials with (potentially) alternative end points and means to detect PMNs and assess their viability after treatment. Given that treatments for metastasis need to be improved and further developed, the field may indeed be ready to take this step.
1. Paget, S. The distribution of secondary growths in cancer of the breast. Lancet 133, 571-573 (1889).

This was the first time the requirement for a suportive microenvironment, or 'fertile soil' in metastatic outgrowth was recognized.

2. Ewing, J. Neoplastic Diseases: A Treatise on Tumours (W. B. Saunders Company, 1928)

3. Fidler, I. J. \& Nicolson, G. L. Organ selectivity for implantation survival and growth of B16 melanoma variant tumor lines. J. Natl Cancer Inst. 57,

1199-1202 (1976).

This study was the first to provide experimental evidence for organotropic metastasis.
4. Hart, I. R. \& Fidler, I. J. Role of organ selectivity in the determination of metastatic patterns of B16 melanoma. Cancer Res. 40, 2281-2287 (1980)

5. Paget, S. The distribution of secondary growths in cancer of the breast. 1889. Cancer Metastasis Rev. 8 98-101 (1989).

6. Psaila, B \& Lyden, D. The metastatic niche: adapting the foreign soil. Nat. Rev. Cancer 9, 285-293 (2009)

7. Kaplan, R. N. et al. VEGFR1-positive haematopoietic bone marrow progenitors initiate the pre-metastatic niche. Nature 438, 820-827 (2005).

This was the first proof-of-principle study demonstrating the existence of and stepwise progression of the PMN.
8. Sleeman, J. P. The lymph node pre-metastatic niche. J. Mol. Med. (Berl.) 93, 1173-1184 (2015).

9. Chin, A. R. \& Wang, S. E. Cancer tills the premetastatic field: mechanistic basis and clinical implications. Clin. Cancer Res. 22, 3725-3733 (2016).

10. Ordonez-Moran, P. \& Huelsken, J. Complex metastatic niches: already a target for therapy? Curr. Opin. Cell Biol. 31, 29-38 (2014).

11. Quail, D. F. \& Joyce, J. A. Microenvironmental regulation of tumor progression and metastasis. Nat. Med. 19, 1423-1437 (2013).

12. Joyce, J. A \& Pollard, J W. Microenvironmental regulation of metastasis. Nat. Rev. Cancer 9 239-252 (2009). 
13. Shibue, T. \& Weinberg, R. A. Metastatic colonization: settlement, adaptation and propagation of tumor cells in a foreign tissue environment. Semin. Cancer Biol. 21, 99-106 (2011).

14. Weilbaecher, K. N., Guise, T. A. \& McCauley, L. K. Cancer to bone: a fatal attraction. Nat. Rev. Cancer 11, 411-425 (2011).

15. Sleeman, J. P. The metastatic niche and stromal progression. Cancer Metastasis Rev. 31, 429-440 (2012).

16. Wculek, S. K. \& Malanchi, I. Neutrophils fan cancer's flames. EMBO J. 34, 2211-2212 (2015).

17. Woodard, P. K., Dehdashti, F. \& Putman, C. E. Radiologic diagnosis of extrathoracic metastases to the lung. Oncology (Williston Park) 12, 431-438 (1998).

18. Francia, G., Cruz-Munoz, W., Man, S., Xu, P. \& Kerbel, R. S. Mouse models of advanced spontaneous metastasis for experimental therapeutics. Nat. Rev. Cancer 11, 135-141 (2011)

19. Karaca, Z. et al. VEGFR1 expression is related to lymph node metastasis and serum VEGF may be a marker of progression in the follow-up of patients with differentiated thyroid carcinoma. Eur. J. Endocrinol. 164, 277-284 (2011).

20. Hirakawa, S. et al. VEGF-C-induced lymphangiogenesis in sentinel lymph nodes promotes tumor metastasis to distant sites. Blood 109, 1010-1017 (2007).

21. Hirakawa, S. et al. VEGF-A induces tumor and sentine lymph node lymphangiogenesis and promotes lymphatic metastasis. J. Exp. Med. 201, 1089-1099 (2005).

22. Jung, T. et al. CD44v6 dependence of premetastatic niche preparation by exosomes. Neoplasia 11, 1093-1105 (2009)

23. Payen, D., Dupuy, P., Schurando, P. \& Laborde, F. Postoperative enoximone in coronary surgery. Systemic and coronary hemodynamics and regional systolic function. Arch. Mal. Coeur Vaiss. 83, 13-17 (in French) $(1990$

24. Headley, M. B. et al. Visualization of immediate immune responses to pioneer metastatic cells in the lung. Nature 531, 513-517 (2016).

25. Zhang, C. et al. Human CD133-positive hematopoietic progenitor cells initiate growth and metastasis of colorectal cancer cells. Carcinogenesis 35 2771-2777 (2014).

26. Zhang, Y., Davis, C., Ryan, J., Janney, C. \& Pena, M. M. Development and characterization of a reliable mouse model of colorectal cancer metastasis to the liver. Clin. Exp. Metastasis 30, 903-918 (2013).

27. Yang, Z. H., Yang, M., Xiong, H. Z. \& Li, X. N. Role of vascular endothelial growth factor receptor 1-positive hematopoietic progenitor cell clusters in human colorectal carcinoma metastasis. Nan Fang Yi Ke Da Хue Xue Bao 28, 696-699 (in Chinese) (2008).

28. Seubert, B. et al. Tissue inhibitor of metalloproteinases (TIMP)-1 creates a premetastatic niche in the liver through SDF-1/CXCR4-dependent neutrophil recruitment in mice. Hepatology 61 238-248 (2015)

29. Costa-Silva, B. et al. Pancreatic cancer exosomes initiate pre-metastatic niche formation in the liver. Nat. Cell Biol. 17, 816-826 (2015).

30. Melo, S. A. et al. Glypican-1 identifies cancer exosomes and detects early pancreatic cancer. Nature 523, 177-182 (2015).

31. Mundy, G. R. Metastasis to bone: causes, consequences and therapeutic opportunities. Nat. Rev. Cancer 2, 584-593 (2002).

32. Cox, T. R. et al. LOX-mediated collagen crosslinking is responsible for fibrosis-enhanced metastasis. Cancer Res. 73, 1721-1732 (2013).

33. Cox, T. R. et al. The hypoxic cancer secretome induces pre-metastatic bone lesions through lysyl oxidase. Nature 522, 106-110 (2015).

34. Guise, T. A. et al. Evidence for a causal role of parathyroid hormone-related protein in the pathogenesis of human breast cancer-mediated osteolysis. J. Clin. Invest. 98, 1544-1549 (1996).

35. Ara, T. et al. Interleukin- 6 in the bone marrow microenvironment promotes the growth and survival of neuroblastoma cells. Cancer Res. 69, 329-337 (2009).

36. Paule, B. et al. Enhanced expression of interleukin-6 in bone and serum of metastatic renal cell carcinoma. Hum. Pathol. 29, 421-424 (1998)

37. Thomas, R. J. et al. Breast cancer cells interact with osteoblasts to support osteoclast formation. Endocrinology 140, 4451-4458 (1999).
38. Kang, Y. et al. A multigenic program mediating breast cancer metastasis to bone. Cancer Cell 3, 537-549 (2003)

This landmark study defined the breast cancer cell-intrinsic determinants of bone metastasis.

39. Lynch, C C et al MMP-7 promotes prostate cancerinduced osteolysis via the solubilization of RANKL. Cancer Cell 7, 485-496 (2005)

40. Lu, X. et al. ADAMTS1 and MMP1 proteolytically engage EGF-like ligands in an osteolytic signaling cascade for bone metastasis. Genes Dev. 23 1882-1894 (2009).

41. Guise, T. Examining the metastatic niche: targeting the microenvironment. Semin. Oncol. 37 (Suppl. 2), S2-S14 (2010).

42. Dai, J. et al. Bone morphogenetic protein- 6 promotes osteoblastic prostate cancer bone metastases through a dual mechanism. Cancer Res. 65, 8274-8285 (2005).

43. Logothetis, C. J. \& Lin, S. H. Osteoblasts in prostate cancer metastasis to bone. Nat. Rev. Cancer 5, 21-28 (2005).

44. Gaur, T. et al. Canonical WNT signaling promotes osteogenesis by directly stimulating Runx 2 gene expression. J. Biol. Chem. 280, 33132-33140 (2005)

45. Bennett, C. N. et al. Regulation of osteoblastogenesis and bone mass by Wnt10b. Proc. Natl Acad. Sci. USA 102, 3324-3329 (2005).

46. Steeg, P. S., Camphausen, K. A. \& Smith, O. R. Brain metastases as preventive and therapeutic targets. Nat. Rev. Cancer 11, 352-363 (2011).

47. Lyle, L. T. et al. Alterations in pericyte subpopulations are associated with elevated blood-tumor barrier permeability in experimental brain metastasis of breast cancer. Clin. Cancer Res. 22, 5287-5299 (2016).

48. Percy, D. B. et al. In vivo characterization of changing blood-tumor barrier permeability in a mouse model of breast cancer metastasis: a complementary magnetic resonance imaging approach. Invest. Radiol. 46 718-725 (2011).

49. Steeg, P. S. Targeting metastasis. Nat. Rev. Cancer 16 201-218 (2016).

50. Fong, M. Y. et al. Breast-cancer-secreted miR-122 reprograms glucose metabolism in premetastatic niche to promote metastasis. Nat. Cell Biol. 17, 183-194 (2015).

This study was the first to provide evidence of metabolic reprogramming of stromal cells in PMNs through miRNA cargo shuttled by tumour-derived microvesicles.

51. Hoshino, A. et al. Tumour exosome integrins determine organotropic metastasis. Nature 527. 329-335 (2015).

This landmark study demonstrated that organotropic metastasis can be orchestrated in a tumour cell-autonomous manner through exosome-expressed integrins.

52. Hiratsuka, S. et al. MMP9 induction by vascular endothelial growth factor receptor-1 is involved in lung-specific metastasis. Cancer Cell 2, 289-300 (2002)

53. Hiratsuka, S. et al. The S100A8-serum amyloid A3TLR4 paracrine cascade establishes a pre-metastatic phase. Nat. Cell Biol. 10, 1349-1355 (2008). This study demonstrated that chemokines induced in PMNs facilitate metastasis in a TLR4-dependent manner, through effects on local innate immune cells.

54. Shojaei, F. et al. G-CSF-initiated myeloid cell mobilization and angiogenesis mediate tumor refractoriness to anti-VEGF therapy in mouse models. Proc. Natl Acad. Sci. USA 106, 6742-6747 (2009)

55. Melgarejo, E., Medina, M. A., Sanchez-Jimenez, F. \& Urdiales, J. L. Monocyte chemoattractant protein-1: a key mediator in inflammatory processes. Int J. Biochem. Cell Biol. 41, 998-1001 (2009).

56. Lu, Y. et al. Monocyte chemotactic protein-1 (MCP-1) acts as a paracrine and autocrine factor for prostate cancer growth and invasion. Prostate 66, 1311-1318 (2006)

57. Cai, Z. et al. Monocyte chemotactic protein 1 promotes lung cancer-induced bone resorptive lesions in vivo. Neoplasia 11, 228-236 (2009).

58. Loberg, R. D. et al. Targeting CCL2 with systemic delivery of neutralizing antibodies induces prostat cancer tumor regression in vivo. Cancer Res. 67 9417-9424 (2007).

59. Saji, H. et al. Significant correlation of monocyte chemoattractant protein-1 expression with neovascularization and progression of breast carcinoma. Cancer 92, 1085-1091 (2001).

60. Lebrecht, A. et al. Monocyte chemoattractant protein-1 serum levels in patients with breast cancer. Tumour Biol. 25, 14-17 (2004).

61. Qian, B. Z. et al. CCL2 recruits inflammatory monocytes to facilitate breast-tumour metastasis. Nature 475, 222-225 (2011).

62. Sceneay, J. et al. Primary tumor hypoxia recruits CD $11 \mathrm{~b}^{+} / \mathrm{Ly} 6 \mathrm{Cmed} / \mathrm{Ly} 6 \mathrm{G}^{+}$immune suppressor cells and compromises NK cell cytotoxicity in the premetastatic niche. Cancer Res. 72, 3906-3911 (2012).

63. Lu, X. \& Kang, Y. Chemokine (C-C motif) ligand 2 engages CCR2 + stromal cells of monocytic origin to promote breast cancer metastasis to lung and bone. J. Biol. Chem. 284, 29087-29096 (2009).

64. Lu, X. \& Kang, Y. Organotropism of breast cancer metastasis. J. Mammary Gland Biol. Neoplasia 12 153-162 (2007)

65. Granot, Z. et al. Tumor entrained neutrophils inhibit seeding in the premetastatic lung. Cancer Cell 20, 300-314 (2011).

66. Bresnick, A. R., Weber, D. J. \& Zimmer, D. B. S100 proteins in cancer. Nat. Rev. Cancer 15, 96-109 (2015)

67. Lukanidin, E. \& Sleeman, J. P. Building the niche: the role of the $\mathrm{S} 100$ proteins in metastatic growth. Semin. Cancer Biol. 22, 216-225 (2012).

68. Donato, R. et al. Functions of $\mathrm{S} 100$ proteins. Curr. Mol. Med. 13, 24-57 (2013).

69. Hiratsuka, S., Watanabe, A., Aburatani, H. \& Maru, Y. Tumour-mediated upregulation of chemoattractants and recruitment of myeloid cells predetermines lung metastasis. Nat. Cell Biol. 8 1369-1375 (2006).

70. Wong, C. C. et al. Hypoxia-inducible factor 1 is a master regulator of breast cancer metastatic niche formation. Proc. Natl Acad. Sci. USA 108 , 16369-16374 (2011).

71. Wong, C. C. et al. Inhibitors of hypoxia-inducible factor 1 block breast cancer metastatic niche formation and lung metastasis. J. Mol. Med. (Berl.) 90, 803-815 (2012)

72. Erler, J. T. et al. Hypoxia-induced lysyl oxidase is a critical mediator of bone marrow cell recruitment to form the premetastatic niche. Cancer Cell 15, 35-44 (2009).

73. Erler, J. T. et al. Lysyl oxidase is essential for hypoxiainduced metastasis. Nature 440, 1222-1226 (2006).

74. King, H. W., Michael, M. Z. \& Gleadle, J. M. Hypoxic enhancement of exosome release by breast cancer cells. BMC Cancer 12, 421 (2012).

75. Wang, T. et al. Hypoxia-inducible factors and RAB22A mediate formation of microvesicles that stimulate breast cancer invasion and metastasis. Proc. Nat Acad. Sci. USA 111, E3234-E3242 (2014).

76. Gould, S. J. \& Raposo, G. As we wait: coping with an imperfect nomenclature for extracellular vesicles. J. Extracell. Vesicles http://dx.doi.org/10.3402/ jev.v2i0.20389 (2013).

77. Colombo, M., Raposo, G. \& Thery, C. Biogenesis, secretion, and intercellular interactions of exosomes and other extracellular vesicles. Annu. Rev. Cell Dev. Biol. 30, 255-289 (2014).

78. Raposo, G. \& Stoorvogel, W. Extracellular vesicles: exosomes, microvesicles, and friends. J. Cell Biol. 200 373-383 (2013)

79. Peinado, H. et al. Melanoma exosomes educate bone marrow progenitor cells toward a pro-metastatic phenotype through MET. Nat. Med. 18, 883-89 (2012).

This was the first study to demonstrate that exosomes secreted by highly metastatic tumours promote metastasis by permanently educating bon marrow progenitors and recruiting them to PMNs.

80. Ostenfeld, M. S. et al. Cellular disposal of miR23b by RAB27-dependent exosome release is linked to acquisition of metastatic properties. Cancer Res. 74 , 5758-5771 (2014).

81. Peinado, H., Lavotshkin, S. \& Lyden, D. The secreted factors responsible for pre-metastatic niche formation old sayings and new thoughts. Semin. Cancer Biol. 21 139-146 (2011).

82. Valadi, H. et al. Exosome-mediated transfer of mRNAs and microRNAs is a novel mechanism of genetic exchange between cells. Nat. Cell Biol. 9, 654-659 (2007).

83. Balaj, L. et al. Tumour microvesicles contain retrotransposon elements and amplified oncogene sequences. Nat. Commun. 2, 180 (2011). 
84. Skog, J. et al. Glioblastoma microvesicles transport RNA and proteins that promote tumour growth and provide diagnostic biomarkers. Nat. Cell Biol. 10 1470-1476 (2008).

85. Al-Nedawi, K. et al. Intercellular transfer of the oncogenic receptor EGFRvIll by microvesicles derived from tumour cells. Nat. Cell Biol. 10, 619-624 (2008).

86. Ratajczak, J. et al. Embryonic stem cell-derived microvesicles reprogram hematopoietic progenitors: evidence for horizontal transfer of mRNA and protein delivery. Leukemia 20, 847-856 (2006).

87. Janowska-Wieczorek, A. et al. Microvesicles derived from activated platelets induce metastasis and angiogenesis in lung cancer. Int. J. Cancer 113 752-760 (2005)

88. Janowska-Wieczorek, A., Marquez-Curtis, L. A., Wysoczynski, M. \& Ratajczak, M. Z. Enhancing effect of platelet-derived microvesicles on the invasive potential of breast cancer cells. Transfusion 46, 1199-1209 (2006).

89. Cocucci, E., Racchetti, G. \& Meldolesi, J. Shedding microvesicles: artefacts no more. Trends Cell Biol. 19 43-51 (2009)

90. lero, M et al. Tumour-released exosomes and their implications in cancer immunity. Cell Death Differ. 15 80-88 (2008)

91. Ratajczak, J., Wysoczynski, M., Hayek, F., JanowskaWieczorek, A. \& Ratajczak, M. Z. Membrane-derived microvesicles: important and underappreciated mediators of cell-to-cell communication. Leukemia 20 1487-1495 (2006).

92. Grange, C. et al. Microvesicles released from human renal cancer stem cells stimulate angiogenesis and formation of lung premetastatic niche. Cancer Res. 71 5346-5356 (2011).

93. Hood, J. L., San, R. S. \& Wickline, S. A. Exosomes released by melanoma cells prepare sentinel lymph nodes for tumor metastasis. Cancer Res. 71 3792-3801 (2011).

94. Villarroya-Beltri, C., Baixauli, F., Gutierrez-Vazquez, C. Sanchez-Madrid, F. \& Mittelbrunn, M. Sorting it out: regulation of exosome loading. Semin. Cancer Biol. 28, 3-13 (2014).

95. Liu, Y. et al. Tumor exosomal RNAs promote lung pre-metastatic niche formation by activating alveolar epithelial TLR3 to recruit neutrophils. Cancer Cell 30 243-256 (2016)

96. Giles, A. J. et al. Activation of hematopoietic stem/ progenitor cells promotes immunosuppression within the pre-metastatic niche. Cancer Res. 76 1335-1347 (2016).

97. Jian, J. et al. Platelet factor 4 is produced by subsets of myeloid cells in premetastatic lung and inhibits tumor metastasis. Oncotarget http://dx. doi. org/10.18632/oncotarget.9486 (2016).

98. Huang, Y. et al. Pulmonary vascular destabilization in the premetastatic phase facilitates lung metastasis. Cancer Res. 69, 7529-7537 (2009).

This landmark study demonstrated that ANGPT2-, MMP3- and MMP10-dependent pulmonary vascular destabilization is an early event occurring during the pre-metastatic phase, which promotes the extravasation of tumour cells and facilitates lung metastasis.

99. Hiratsuka, S. et al. Primary tumours modulate innate immune signalling to create pre-metastatic vascular hyperpermeability foci. Nat. Commun. 4, 1853 (2013)

100. Hiratsuka, S. et al. Endothelial focal adhesion kinase mediates cancer cell homing to discrete regions of the lungs via E-selectin up-regulation. Proc. Natl Acad. Sci. USA 108, 3725-3730 (2011).

101. Yan, H. H. et al. Gr-1+CD $11 \mathrm{~b}^{+}$myeloid cells tip the balance of immune protection to tumor promotion in the premetastatic lung. Cancer Res. 70, 6139-6149 (2010).

102. Gupta, G. P. et al. Mediators of vascular remodelling co-opted for sequential steps in lung metastasis. Nature 446, 765-770 (2007)

103. Padua, D. et al. TGF $\beta$ primes breast tumors for lung metastasis seeding through angiopoietin-like 4 Cell 133, 66-77 (2008).

104. Jean, C. et al. Inhibition of endothelial FAK activity prevents tumor metastasis by enhancing barrier function. J. Cell Biol 204, 247-263 (2014).

105. Kim, S. et al. Carcinoma-produced factors activate myeloid cells through TLR2 to stimulate metastasis Nature 457, 102-106 (2009)

106. Bos, P. D. et al. Genes that mediate breast cancer metastasis to the brain. Nature 459, 1005-1009 (2009).
107. Sevenich, L. et al. Analysis of tumour- and stromasupplied proteolytic networks reveals a brainmetastasis-promoting role for cathepsin S. Nat. Cell Biol. 16, 876-888 (2014).

108. Gay, L. J. \& Felding-Habermann, B. Contribution of platelets to tumour metastasis. Nat. Rev. Cancer 11 123-134 (2011).

109. Kuderer, N. M., Ortel, T. L. \& Francis, C. W. Impact of venous thromboembolism and anticoagulation on cancer and cancer survival. J. Clin. Oncol. 27, 4902-4911 (2009).

110. Im, J. H. et al. Coagulation facilitates tumor cell spreading in the pulmonary vasculature during early metastatic colony formation. Cancer Res. 64 8613-8619 (2004)

111. Gil-Bernabe, A. M et al. Recruitment of monocytes/ macrophages by tissue factor-mediated coagulation is essential for metastatic cell survival and premetastatic niche establishment in mice. Blood 119, 3164-3175 (2012).

112. Labelle, M., Begum, S. \& Hynes, R. O. Platelets guide the formation of early metastatic niches. Proc. Nat Acad. Sci. USA 111, E3053-E3061 (2014).

113. Hansen, M. T. et al. A link between inflammation and metastasis: serum amyloid $\mathrm{A} 1$ and $\mathrm{A} 3$ induce metastasis, and are targets of metastasis-inducing S100A4. Oncogene 34, 424-435 (2015).

114. Mauti, L. A. et al. Myeloid-derived suppressor cells are implicated in regulating permissiveness for tumor metastasis during mouse gestation. J. Clin. Invest. 121, 2794-2807 (2011).

115. Sharma, S. K. et al. Pulmonary alveolar macrophages contribute to the premetastatic niche by suppressing antitumor T cell responses in the lungs. J. Immunol. 194, 5529-5538 (2015).

116. Malanchi, l. et al. Interactions between cancer stem cells and their niche govern metastatic colonization. Nature 481, 85-89 (2012)

This landmark study demonstrated that a subpopulation of cancer stem cells is responsible for metastatic colonization and that this process depends on signals provided by the stromal niche.

117. Kudo, A. Periostin in fibrillogenesis for tissue regeneration: periostin actions inside and outside the cell. Cell. Mol. Life Sci. 68, 3201-3207 (2011).

118. Fukuda, K. et al. Periostin is a key niche component for wound metastasis of melanoma. PLOS ONE 10 e0129704 (2015)

119. Wang, Z. et al. Periostin promotes immunosuppressive premetastatic niche formation to facilitate breast tumour metastasis. J. Pathol. 239, 484-495 (2016)

120. Gao, D. et al. Myeloid progenitor cells in the premetastatic lung promote metastases by inducing mesenchymal to epithelial transition. Cancer Res. 72 1384-1394 (2012)

121. Egeblad, M. \& Werb, Z. New functions for the matrix metalloproteinases in cancer progression. Nat. Rev. Cancer 2, 161-174 (2002).

122. Cameron, J. D. Skubitz, A. P. \& Furcht, L. T. Type IV collagen and corneal epithelial adhesion and migration. Effects of type IV collagen fragments and synthetic peptides on rabbit corneal epithelial cell adhesion and migration in vitro. Invest. Ophthalmol. Vis. Sci. 32, 2766-2773 (1991).

123. Shahan, T. A., Fawzi, A., Bellon, G., Monboisse, J. C. $\&$ Kefalides, N. A. Regulation of tumor cell chemotaxis by type IV collagen is mediated by a $\mathrm{Ca}^{2+}$-dependent mechanism requiring $\mathrm{CD} 47$ and the integrin $\alpha_{v} \beta_{3}$. J. Biol. Chem. 275, 4796-4802 (2000)

124. Kessenbrock, K., Plaks, V. \& Werb, Z. Matrix metalloproteinases: regulators of the tumo microenvironment. Cell 141, 52-67 (2010)

125. van Deventer, H. W. et al. C-C chemokine receptor 5 on stromal cells promotes pulmonary metastasis. Cancer Res. 65, 3374-3379 (2005)

126. van Deventer, H. W. et al. C-C chemokine receptor 5 on pulmonary fibrocytes facilitates migration and promotes metastasis via matrix metalloproteinase 9 . Am. J. Pathol. 173, 253-264 (2008).

127. Canesin, G. et al. Lysyl oxidase-like 2 (LOXL2) and E47 EMT factor: novel partners in E-cadherin repression and early metastasis colonization. Oncogene 34 951-964 (2015)

128. Engler, A. J., Humbert, P. O., Wehrle-Haller, B. \& Weaver, V. M. Multiscale modeling of form and function. Science 324, 208-212 (2009).

129. Krieg, M. et al. Tensile forces govern germ-layer organization in zebrafish. Nat. Cell Biol. 10, 429-436 (2008).
130. Ronnov-Jessen, L. \& Bissell, M. J. Breast cancer by proxy: can the microenvironment be both the cause and consequence? Trends Mol. Med. 15, 5-13 (2009).

131. Levental, K. R. et al. Matrix crosslinking forces tumor progression by enhancing integrin signaling. Cell 139 891-906 (2009)

132. Goetz, J. G. et al. Biomechanical remodeling of the microenvironment by stromal caveolin-1 favors tumo invasion and metastasis. Cell 146, 148-163 (2011).

133. Aguado, B. A. et al. Extracellular matrix mediators of metastatic cell colonization characterized using scaffold mimics of the pre-metastatic niche. Acta Biomater. 33 13-24 (2016)

134. White, E. S. \& Muro, A. F. Fibronectin splice variants: understanding their multiple roles in health and disease using engineered mouse models. IUBMB Life 63, 538-546 (2011)

135. Papaspyridonos, M. et al. Id 1 suppresses anti-tumour immune responses and promotes tumour progression by impairing myeloid cell maturation. Nat. Commun 6, 6840 (2015)

136. Kowanetz, M. et al. Granulocyte-colony stimulating factor promotes lung metastasis through mobilization of Ly6G ${ }^{+}$Ly6C ${ }^{+}$granulocytes. Proc. Natl Acad. Sci. USA 107, 21248-21255 (2010)

137. Casbon, A. J. et al. Invasive breast cancer reprograms early myeloid differentiation in the bone marrow to generate immunosuppressive neutrophils. Proc. Natl Acad. Sci. USA 112, E566-E575 (2015).

138. Bergers, G. et al. Matrix metalloproteinase- 9 triggers the angiogenic switch during carcinogenesis. Nat. Cell Biol. 2, 737-744 (2000).

139. Ahn, G. O. \& Brown, J. M. Matrix metalloproteinase-9 is required for tumor vasculogenesis but not for angiogenesis: role of bone marrow-derived myelomonocytic cells. Cancer Cell 13, 193-205 (2008).

140. Wu, C. F. et al. The lack of type I interferon induces neutrophil-mediated pre-metastatic niche formation in the mouse lung. Int. J. Cancer 137, 837-847 (2015).

141. Zaidi, M. R. \& Merlino, G. The two faces of interferon- $\gamma$ in cancer. Clin. Cancer Res. 17, 6118-6124 (2011).

142. Wculek, S. K. \& Malanchi, I. Neutrophils support lung colonization of metastasis-initiating breast cancer cells. Nature 528, 413-417 (2015)

143. Benito-Martin, A., Di Giannatale, A., Ceder, S. \& Peinado, H. The new deal: a potential role for secreted vesicles in innate immunity and tumor progression. Front. Immunol. 6, 66 (2015).

144. Moses, W. W. Fundamental limits of spatial resolution in PET. Nucl. Instrum. Methods Phys. Res. A 648 (Suppl. 1), S236-S240 (2011).

145. Marom, E. M., Sarvis, S., Herndon, J. E. II \& Patz, E. F. Jr. T1 lung cancers: sensitivity of diagnosis with fluorodeoxyglucose PET. Radiology 223 453-459 (2002)

146. James, K. et al. Measuring response in solid tumors: unidimensional versus bidimensional measurement. J. Natl Cancer Inst. 91, 523-528 (1999).

147. Diaz-Cano, S. J. Tumor heterogeneity: mechanisms and bases for a reliable application of molecular marker design. Int. J. Mol. Sci. 13, 1951-2011 (2012).

148. leni, A., Giuffre, G., Adamo, V. \& Tuccari, G. Prognostic impact of CD133 immunoexpression in node-negative invasive breast carcinomas. Anticancer Res. 31 1315-1320 (2011)

149. Jain, S. et al. Incremental increase in VEGFR1 hematopoietic progenitor cells and VEGFR2 endothelial progenitor cells predicts relapse and lack of tumor response in breast cancer patients. Breast Cancer Res. Treat. 132, 235-242 (2012).

150. Kosaka, Y. et al. Identification of the high-risk group for metastasis of gastric cancer cases by vascular endothelial growth factor receptor-1 overexpression in peripheral blood. Br. J. Cancer 96, 1723-1728 (2007)

151. Xu, W. W. et al. Targeting VEGFR 1 - and VEGFR2-expressing non-tumor cells is essential for esophageal cancer therapy. Oncotarget 6 1790-1805 (2015)

152. Zhang, W. et al. Myeloid clusters are associated with a pro-metastatic environment and poor prognosis in smoking-related early stage non-small cell lung cancer. PLOS ONE 8, e65121 (2013).

153. Zhang, W. et al. CD8+ T-cell immunosurveillance constrains lymphoid premetastatic myeloid cell accumulation. Eur. J. Immunol. 45, 71-81 (2015).

154. Deng, J. et al. S1PR1-STAT3 signaling is crucial for myeloid cell colonization at future metastatic sites. Cancer Cell 21, 642-654 (2012). 
This study demonstrated that myeloid cells depend on S1PR1-STAT3 signalling to participate in PMN formation.

155. Pala, S. et al. Prognostic significance of neutrophilic infiltration in benign lymph nodes in patients with muscle-invasive bladder cancer. Eur. Urol. http:// dx.doi.org/10.1016/j.euf.2016.03.003 (2016).

156. Cicatiello, V. et al. Powerful anti-tumor and antiangiogenic activity of a new anti-vascular endothelial growth factor receptor 1 peptide in colorectal cancer models. Oncotarget 6, 10563-10576 (2015).

157. Fraga, C. A. et al. A high HIF-1 $\alpha$ expression genotype is associated with poor prognosis of upper aerodigestive tract carcinoma patients. Oral Oncol. 48, 130-135 (2012).

158. Otto, B. et al. Molecular changes in pre-metastatic lymph nodes of esophageal cancer patients. PLOS ONE 9, e102552 (2014)

159. Wakisaka, N. et al. Primary tumor-secreted lymphangiogenic factors induce pre-metastatic lymphvascular niche formation at sentinel lymph nodes in oral squamous cell carcinoma. PLOS ONE 10 e0144056 (2015).

160. Vered, M. et al. Factors associated with collagen metabolism in the lymph node pre-metastatic niche in oral cancer. Oral Surg. Oral Med. Oral Pathol. Oral Radiol. 120, e155-e156 (2015).

161. Pal, S. K. \& Figlin, R. A. Targeted therapies: pazopanib: carving a niche in a crowded therapeutic landscape. Nat. Rev. Clin. Oncol. 7, 362-363 (2010).

162. US National Library of Medicine. ClinicalTrials.gov https://clinicaltrials.gov/ct2/show/NCT01832259 (2016).

163. Torrano, V. et al. Vesicle-MaNiA: extracellular vesicles in liquid biopsy and cancer. Curr. Opin. Pharmacol. 29, 47-53 (2016).

164. Gold, B., Cankovic, M., Furtado, L. V., Meier, F. \& Gocke, C. D. Do circulating tumor cells, exosomes, and circulating tumor nucleic acids have clinical utility? A report of the association for molecular pathology. J. Mol. Diagn. 17, 209-224 (2015).

165. Zhou, W. et al. Cancer-secreted miR-105 destroys vascular endothelial barriers to promote metastasis. Cancer Cell 25, 501-515 (2014).

166. Hu, L., Wickline, S. A. \& Hood, J. L. Magnetic resonance imaging of melanoma exosomes in lymph nodes. Magn. Reson. Med. http://dx.doi.org/10.1002/ mrm. 25376 (2014).

167. Shokeen, M. et al. Molecular imaging of very late antigen-4 (alpha4beta1 integrin) in the premetastatic niche. J. Nucl. Med. 53, 779-786 (2012).

168. Soodgupta, D. et al. Very late antigen-4 $\left(\alpha_{4} \beta_{1}\right.$ Integrin $)$ targeted PET imaging of multiple myeloma. PLOS ONE 8, e55841 (2013).

169. Zhu, L. et al. Label-free quantitative detection of tumor-derived exosomes through surface plasmon resonance imaging. Anal. Chem. 86, 8857-8864 (2014).

170. Joo, Y. N et al. P2Y2R activation by nucleotides released from the highly metastatic breast cancer cell MDA-MB-231 contributes to pre-metastatic niche formation by mediating lysyl oxidase secretion, collagen crosslinking, and monocyte recruitment. Oncotarget 5, 9322-9334 (2014).

171. Liu, Z. et al. Protein tyrosine phosphatase receptor type $\mathrm{O}$ expression in the tumor niche correlates with reduced tumor growth, angiogenesis, circulating tumor cells and metastasis of breast cancer. Oncol Rep 33, 1908-1914 (2015)

172. Ling, X. et al. The CXCR4 antagonist AMD3465 regulates oncogenic signaling and invasiveness in vitro and prevents breast cancer growth and metastasis in vivo. PLoS ONE 8, e58426 (2013).

173. Kirsch, M., Schackert, G. \& Black, P. M. Angiogenesis, metastasis, and endogenous inhibition. J. Neurooncol. 50, 173-180 (2000)

174. Bissell, M. J. \& Hines, W. C. Why don't we get more cancer? A proposed role of the microenvironment in restraining cancer progression. Nat. Med. 17 320-329 (2011)

175. Ghajar, C. M. Metastasis prevention by targeting the dormant niche. Nat. Rev. Cancer 15, 238-247 (2015)

176. Baillargeon, J. \& Rose, D. P. Obesity, adipokines, and prostate cancer (review). Int. J. Oncol. 28, 737-745 (2006).

177. Mistry, T., Digby, J. E., Desai, K. M. \& Randeva, H. S. Obesity and prostate cancer: a role for adipokines. Eur. Urol. 52, 46-53 (2007)
178. Ribeiro, R. J. et al. Tumor cell-educated periprostatic adipose tissue acquires an aggressive cancerpromoting secretory profile. Cell. Physiol. Biochem. 29, 233-240 (2012).

179. Nieman, K. M. et al. Adipocytes promote ovarian cancer metastasis and provide energy for rapid tumor growth. Nat. Med. 17, 1498-1503 (2011).

180. Thaker, P. H. et al. Chronic stress promotes tumor growth and angiogenesis in a mouse model of ovarian carcinoma. Nat. Med. 12, 939-944 (2006).

181. Cox, T. R. \& Erler, J. T. Remodeling and homeostasis of the extracellular matrix: implications for fibrotic diseases and cancer. Dis. Model. Mech. 4, 165-178 (2011).

182. Lim, C. et al. Hepatic ischemia-reperfusion increases circulating bone marrow-derived progenitor cells and tumor growth in a mouse model of colorectal liver metastases. J. Surg. Res. 184, 888-897 (2013).

183. Govaert, K. M. et al. Hypoxia after liver surgery imposes an aggressive cancer stem cell phenotype on residual tumor cells. Ann. Surg. 259, 750-759 (2014).

184. Jiao, S. F. et al. Inhibition of tumor necrosis factor alpha reduces the outgrowth of hepatic micrometastasis of colorectal tumors in a mouse model of liver ischemia-reperfusion injury. J. Biomed. Sci. 21, 1 (2014).

185. van der Bilt, J. D. et al. Ischemia/reperfusion accelerates the outgrowth of hepatic micrometastases in a highly standardized murine model. Hepatology 42, 165-175 (2005).

186. Retsky, M. et al. Reduction of breast cancer relapses with perioperative non-steroidal anti-inflammatory drugs: new findings and a review. Curr. Med. Chem 20, 4163-4176 (2013).

187. Jain, R. K. Normalization of tumor vasculature: an emerging concept in antiangiogenic therapy. Science 307, 58-62 (2005)

188. Scadden, D. T. Nice neighborhood: emerging concepts of the stem cell niche. Cell 157, 41-50 (2014).

189. Bergers, G. \& Hanahan, D. Modes of resistance to anti-angiogenic therapy. Nat. Rev. Cancer 8, 592-603 (2008).

190. Janni, W. et al. Persistence of disseminated tumor cells in the bone marrow of breast cancer patients predicts increased risk for relapse - a European pooled analysis. Clin. Cancer Res. 17, 2967-2976 (2011)

191. Naumov, G. N. et al. Persistence of solitary mammary carcinoma cells in a secondary site: a possible contributor to dormancy. Cancer Res. 62 2162-2168 (2002)

192. Suzuki, M., Mose, E. S., Montel, V. \& Tarin, D. Dormant cancer cells retrieved from metastasis-free organs regain tumorigenic and metastatic potency. Am. J. Pathol. 169, 673-681 (2006).

193. Gao, H. et al. The BMP inhibitor Coco reactivates breast cancer cells at lung metastatic sites. Cell 150 764-779 (2012). This landmark study screening for modifiers of metastatic dormancy identified organ-specific BMP signalling as a microenvironmental suppressor of metastasis.

194. Bragado, P. et al. TGF-beta2 dictates disseminated tumour cell fate in target organs through TGF-beta-RIII and p38alpha/beta signalling. Nat. Cell Biol. 15, 1351-1361 (2013).

195. Kobayashi, A. et al. Bone morphogenetic protein 7 in dormancy and metastasis of prostate cancer stem-like cells in bone. J. Exp. Med. 208, 2641-2655 (2011)

196. Ghajar, C. M. et al. The perivascular niche regulates breast tumour dormancy. Nat. Cell Biol. 15, 807-817 (2013).

This is an elegant dissection of the role of vascular niches in tumour dormancy, demonstrating that stable microvasculature is required to maintain dormancy.

197. Kobayashi, H. et al. Angiocrine factors from Aktactivated endothelial cells balance self-renewal and differentiation of haematopoietic stem cells. Nat. Cell Biol. 12, 1046-1056 (2010)

198. Franses, J. W., Drosu, N. C., Gibson, W. J., Chitalia, V. C. \& Edelman, E. R. Dysfunctional endothelial cells directly stimulate cancer inflammation and metastasis. Int. J. Cancer 133. 1334-1344 (2013)

199. Klein, C. A. Parallel progression of primary tumours and metastases. Nat. Rev. Cancer 9, 302-312 (2009).

200. Friedl, P. \& Wolf, K. Tumour-cell invasion and migration: diversity and escape mechanisms. Nat. Rev. Cancer 3, 362-374 (2003)
201. Wong, S. Y. \& Hynes, R. O. Lymphatic or hematogenous dissemination: how does a metastatic tumor cell decide? Cell Cycle 5 812-817 (2006)

202. Hall, C. L. et al. Type I collagen receptor $\left(\alpha_{2} \beta_{1}\right)$ signaling promotes prostate cancer invasion through RhoC GTPase. Neoplasia 10, 797-803 (2008).

203. Zhou, B. et al. Integrin $\alpha_{3} \beta_{1}$ can function to promote spontaneous metastasis and lung colonization of invasive breast carcinoma. Mol. Cancer Res. 12, 143-154 (2014)

204. Mori, Y. et al. Anti- $\alpha_{4}$ integrin antibody suppresses the development of multiple myeloma and associated osteoclastic osteolysis. Blood 104, 2149-2154 (2004).

205. Clezardin, P. Integrins in bone metastasis formation and potential therapeutic implications. Curr. Cancer Drug Targets 9, 801-806 (2009)

206. Schneider, J. G., Amend, S. R. \& Weilbaecher, K. N. Integrins and bone metastasis: integrating tumor cell and stromal cell interactions. Bone 48, 54-65 (2011).

207. Tome, Y. et al. High lung-metastatic variant of human osteosarcoma cells, selected by passage of lung metastasis in nude mice, is associated with increased expression of $\alpha_{2} \beta_{3}$ integrin. Anticancer Res. 33 , 3623-3627 (2013).

208. Hatano, M. et al. Cadherin-11 regulates the metastasis of Ewing sarcoma cells to bone. Clin. Exp. Metastasis 32, 579-591 (2015).

209. Muller, A. et al. Involvement of chemokine receptors in breast cancer metastasis. Nature 410, 50-56 (2001).

210. Cheng, H. C., Abdel-Ghany, M., Elble, R. C. \& Pauli, B. U. Lung endothelial dipeptidyl peptidase IV promotes adhesion and metastasis of rat breast cancer cells via tumor cell surface-associated fibronectin. J. Biol. Chem. 273, 24207-24215 (1998)

211. Petretti, T., Kemmner, W., Schulze, B. \& Schlag, P. M Altered mRNA expression of glycosyltransferases in human colorectal carcinomas and liver metastases. Gut 46, 359-366 (2000).

212. Yasmin-Karim, S., King, M. R., Messing, E. M. \& Lee, Y. F. E-Selectin ligand-1 controls circulating prostate cancer cell rolling/adhesion and metastasis. Oncotarget 5, 12097-12110 (2014).

213. Dimitroff, C. J. et al. Identification of leukocyte E-selectin ligands, P-selectin glycoprotein ligand-1 and E-selectin ligand-1, on human metastatic prostate tumor cells. Cancer Res. 65, 5750-5760 (2005).

214. Kang, Y. et al. Breast cancer bone metastasis mediated by the Smad tumor suppressor pathway. Proc. Natl Acad. Sci. USA 102, 13909-13914 (2005).

215. Minn, A. J. et al. Genes that mediate breast cancer metastasis to lung. Nature 436, 518-524 (2005).

216. Narita, T. et al. Induction of E-selectin expression on vascular endothelium by digestive system cancer cells. J. Gastroenterol. 31, 299-301 (1996).

\section{Acknowledgements}

The authors gratefully acknowledge support from the following funding sources: the US National Cancer Institute (CA169538 to D.L., M.J.B. and H.P. and CA169416 to D.L. and H.P.), the US Department of Defense (W81XWH-13-1-0427 to Y.K., D.L. and J.B., W81 XWH-13-1-0249 and W81XWH-14-1-0199 to D.L.), the Hartwell Foundation, the Manning Foundation, the Sohn Foundation, the STARR Consortium, the POETIC Consortium, the Paduano Foundation, Alex's Lemonade Stand Foundation, the Champalimaud Foundation, the 5th District AHEPA Cancer Research Foundation (all to D.L.) and the Daedalus Fund (Weill Cornell Medicine, to D.L and H.Z). H.P. is supported by grants from MINECO (SAF2014-54541-R), ATRES-MEDIA - AXA, Asociaciōn Española Contra el Cáncer, WHRI Academy and Worldwide Cancer Research. A.H. is sup ported by a Susan Komen Foundation For the Cure Fellowship. J.T.E. is supported by a Novo Nordisk Foundation Hallas Møller stipend. G.R. is supported by a Peter Oppenheimer Fellowship, awarded by the American Portuguese Biomedical Research Fund. C.M.G is supported by a US Department of Defense Breast Cancer Research Program Era of Hope Scholar Award (W81XWH-15-1-0201), the US National Cancer Institute (CA193461-01), the National Breast Cancer Coalition's Artemis Project and the Pink Gene Foundation.

Competing interests statement

The authors declare no competing interests. 